\title{
DROSHA and DICER RNA products control BMI1- dependent transcriptional repression at DNA damage sites
}

\section{Sofia Francia ( $\sim$ sofia.francia@igm.cnr.it)}

Istituto di Genetica Molecolare https://orcid.org/0000-0002-4578-9442

llaria Capozzo

Istituto di Genetica Molecolare

\section{Stefania Modafferi}

Istituto di Genetica Molecolare https://orcid.org/0000-0002-5669-860X

\section{Fabio lannelli}

The FIRC Institute of Molecular Oncology, IFOM, Milano https://orcid.org/0000-0002-2777-1417

\section{Ubaldo Gioia}

IFOM - the FIRC Institute of Molecular Oncology https://orcid.org/0000-0002-4993-5283

\section{Fabrizio d'Adda di Fagagna}

FIRC Institute of Molecular Oncology https://orcid.org/0000-0002-9603-5966

\section{Article}

Keywords:

Posted Date: January 18th, 2022

DOI: https://doi.org/10.21203/rs.3.rs-1214613/v1

License: (1) This work is licensed under a Creative Commons Attribution 4.0 International License.

Read Full License 


\title{
DROSHA and DICER RNA products control BMI1-dependent transcriptional repression at DNA damage sites
}

Ilaria Capozzo ${ }^{1 \S}$, Stefania Modafferi ${ }^{1}$, Fabio Iannelli ${ }^{2}$, Ubaldo Gioia ${ }^{2}$, Fabrizio d'Adda di Fagagna ${ }^{1,2}$ and Sofia Francia ${ }^{1,2,3}$

1. Istituto di Genetica Molecolare, CNR - Consiglio Nazionale delle Ricerche, Pavia, 27100, Italy.

2. IFOM Foundation - The FIRC Institute of Molecular Oncology Foundation, Milan, 20139, Italy.

3. Correspondence to: sofia.francia@igm.cnr.it

$\S$. Present address: IEO, Istituto Europeo di Oncologia IRCCS, Via Ripamonti 435, 20141, Milan, Italy.

\begin{abstract}
Genome integrity is safeguarded by the DNA damage response (DDR). Transcriptional modulation of genes around DNA double-strand breaks (DSBs) is important for DNA repair. It has been shown that DSBs repress transcription of DSB-bearing genes in an ATM- and PRC1-dependent manner. However, DSB also induce local de novo transcription of non-coding RNA, which are processed by DROSHA and DICER into small DNA-damage-response RNA (DDRNA). Here we reconcile these apparently contrasting observations by showing that DROSHA and DICER inactivation prevents transcriptional repression of DSB-bearing genes by reducing PRC1 recruitment to DSB and consequent H2A-K119 chromatin ubiquitination. Indeed, DDRNAs generated at DSB associate with the PRC1 component BMI1 and inhibition of DDRNA function with antisense oligonucleotides is sufficient to reduce damage-induce transcriptional silencing in cis genes (DISC). We propose that DROSHA, DICER and DDRNAs control DISC at genomic lesion sites by favoring PRC1-driven chromatin ubiquitination.
\end{abstract}

\section{Introduction}

Generation of small non-coding RNAs at DNA damage sites has been observed in 
several organisms and genomic contexts [1]; [2]; [3]; [4]; [5]. In mammalian cells, we and others have observed that a double strand break (DSB) induces de novo transcription of non-coding RNA [5-8]. Basically, DSBs recruit the DNA damage sensor MRE11RAD50-NBS1 (MRN) complex, that has the ability to melt, unwind DNA double helix[9], thus loading RNA polymerase II with components of the Pre Initiation Complex (PIC), similarly to what occurs at a canonical promoter [10]. This causes de novo transcription from DNA ends of RNA molecules defined damage-induced longnon coding RNA (dilncRNA) that are then processed by DROSHA and DICER, physically recruited to DSB [11-14]. DilncRNA processing by DROSHA and DICER generates small DNA damage RNA (DDRNA) that, together with their precursor, promotes the activation of the DNA damage response (DDR) signaling pathway [1519]. Similar events occur at dysfunctional telomeres [20, 21]. DROSHA has also been proposed to mediate DNA:RNA hybrid formation following DNA damage at transcribed endogenous sites, supporting the notion that the RNAi machinery is active at DSB[22].

We and others have however also shown that pre-existing transcription of coding genes in proximity of a DSB is actively repressed through different mechanisms, [23-27]. Specifically, kinase activity of ATM, an apical DDR kinase, has been causally involved in DISC [23, 24, 26, 28]. ATM phosphorylation of Brahma-related gene-1 (BRG1) or hbrm-associated factor 180 (BAF180), a Polybromo-associated BAF (PBAF) remodeling complex's subunit, has been reported to be important for DSB-induced transcriptional silencing [25]. Polycomb group complexes 1 and 2 (PRC1 and PRC2) have been shown to be required for DSB-induced transcriptional silencing [25] and ATM phosphorylation of ENL, a component of the elongating transcriptional machinery, stimulates its association with the PRC1 subunit, BMI1. This interaction promotes the accumulation of BMI1 at actively-transcribed genes flanking a DSB and the consequent PRC1-mediated mono-ubiquitylation of histone H2A on lysine 119 (ubH2A-K119), thus inhibiting transcription at DSB [29]. Indeed, BMI-mediated ub H2A-K119 promotes DNA repair [30].

Therefore, DSBs induce both a de novo transcription of non-coding RNAs followed by processing by component of the RNAi machinery, and an active repression of preexisting coding transcription of DSB-bearing canonical genes. How these two apparently opposite transcriptional events influence each other remains unknown.

By combining molecular and cellular biology techniques here we show that DROSHA 
and DICER RNA products control DSB-induced transcriptional silencing of canonical genes. Moreover, the pharmacological stimulation of DICER activity by enoxacin, enhances DISC. Mechanistically, we show that DROSHA and DICER stimulate BMI1 recruitment and H2A-K119 ubiquitination, which are required for DISC. Importantly, BMI1 associates with DDRNAs upon DSB induction and inhibitory antisense oligonucleotide (ASO) designed against dilncRNA and DDRNA formed at DSB prevent DISC, supporting a specific role for DDRNA products in transcriptional silencing.

\section{Results}

\section{Transcriptional repression of a reporter gene by DSB requires DICER.}

In Drosophila melanogaster and in Neurospora crassa cells, DNA-damage induced and RNAi-dependent small ncRNAs have been proposed to act as endogenous siRNA silencing genes encoded by the region flanking DNA lesions[2, 31]. We thus hypothesized that DDRNAs may act in a similar fashion, repressing transcription of active genes flanking DSB in mammalian cells and we therefore investigated the potential contribution of DROSHA and DICER and of their RNA products DDRNAs, in DSB-induced transcription silencing in cis (DISC) as recently defined in [28]. To test this hypothesis, we initially took advantage of an engineered cellular system previously used to demonstrate DISC: the U2OS-2-6-3 cells [23]. In this system, a cluster of DSBs can be induced upstream of an inducible reporter gene [23] which can be visualized in the cell thanks to the genomic integration of hundreds of copies of Lac operator ( $\mathrm{LacO}$ ) and the expression of the FokI endonuclease fused with a Lac inhibitor (Lac I) and the fluorescent mCherry tag. This allows the generation multiple DSBs at the LacO repeats and the cytological visualization of the damaged locus by Cherry signal accumulation. Nuclease-deficient D450A FokI mutant (FokI D450A) is used as negative control. Downstream to the cleavable repeats, cells bear an inducible gene encoding for a CFPtagged peroxisomal targeting peptide (CFP-SKL) bearing 24 stem-loop structures which bind the yellow fluorescent protein (YFP)-tagged MS2 viral protein, also expressed under doxycycline control (for a schematic description of the locus see Figure 1A and [23]). Thus, YFP-MS2 proteins bind to nascent CFP-SKL transcripts allowing a rapid visualization of RNA generation and the study of DISC [23]. In the same system, the precise quantification of nascent transcripts can be achieved by RT-qPCR analysis at the CFP-SKL gene. As previously reported[23], we observed that expression of FOK 
WT, but not of D450A FOK mutant isoform (uncut condition), reduces of 40-50\% CFPSKL gene transcripts levels (Figure 1A) as detected by RT-qPCR, and that ATM knockdown prevents DISC in this system (KD - Figure S1A). Here, we tested the impact of DROSHA and DICER inactivation on DISC. By RT-qPCR analysis of the CFP-SKL transcript, we observed that DICER inactivation almost completely abolishes DISC in a manner quantitatively similar to ATM inactivation (Figure 1B). In this setting DROSHA inactivation instead, despite similar knockdown efficiency (Figure S1B) shows a modest impact on DISC (Fig 1A) - this is likely due to the fact that CFP-SKL reporter transcripts are structured in multiple stem-loops that DROSHA can recognize and cleave them thus compounding the interpretation of DROSHA role in DISC on this construct. Indeed, stem-loop RNA labeling can affect mRNA processing [32]. For this reason, in this cellular setting, we decided to focus on the role of DICER only.

Next, we analyzed the YFP-MS2 fluorescent signal in cut and uncut cells, upon DICER or ATM KD at single-cell level. Upon damage in control cells, we observed an evident reduction of YFP-MS2 focal signal confirming DISC (Figure 1B and C). Analyses of MS2-YFP fluorescent signals in DICER- or ATM-inactivated cells revealed a more persistent MS2-YFP fluorescent signal, indicating impaired DISC (Figure 1B and C). This result confirms that DICER, similarly to ATM, is indeed required for DISC. Immunofluorescence (IF) against $\gamma \mathrm{H} 2 \mathrm{AX}$ demonstrated similar amounts of DNA damage in all cut conditions, as previously observed in other cellular systems $[6,15,18]$ whereas, consistently with previous observations in this system [23, 25, 29, 33], ATM inactivation slightly decreases it (Figure 1D).

This set of data indicates that DICER, similarly to ATM, is required for transcriptional silencing of genes next to damaged chromatin. Importantly, the CFP reporter transcript is expressed by an exogenous integrated construct and a computational analysis confirmed that it is not targeted by endogenous miRNAs, making DDRNA rather than miRNAs the likely DICER products responsible for the observed effects.

\section{DROSHA and DICER control DISC at endogenous genes caused by individual DSB.}

To extend and strengthen our conclusions, we took advantage of the DIvA cellular system [34]. In these cells, the restriction enzyme AsiSI is expressed under the control of a modified version of the Estrogen-Receptor binding domain, thus its nuclear localization and DNA cleavage activity can be induced by the addition of 4Hydroxytamoxifen to the medium[34, 35]. By cap analysis of gene expression (CAGE) 
in this cellular system, we previously reported that the DISC takes place also at endogenous genes upon single DSB generation [26]. We and others observed that Translin/Trax complex can compensate for DICER's activity loss in different circumstances, but this do not occur if also DROSHA is KD $[6,36]$. For this reason, throughout the study we preferentially used concomitant knockdown (co-KD) of DROSHA and DICER as a more efficient approach to study the impact of DDRNA on DISC. In the well-characterized DIvA cellular system, we performed RNA sequencing by pely $A$ capture in cut and uncut conditions comparing control siRNA-transfected cells to DICER and DROSHA co-KD or ATM KD. In our computational analyses we took under consideration the expression level of the 196 genes previously reported as cut in AsiSI sites within or adjacent $( \pm 2 \mathrm{~Kb})$ to gene bodies [26]. Among these we focused on the 63 genes that were downregulated in control siRNA (siCTRL) samples upon cut. Relative differential expression analyses of the 63 genes revealed that DICER and DROSHA co-KD, similarly to ATM, reduces the ability of cells to induce DISC of endogenous DSB-bearing genes as indicated by a shift towards zero of the $\log 2$ of the fold change between the cut expression level versus the uncut one (Figure 2A). This unbiased genome-wide analysis indicates that DROSHA and DICER control DISC at endogenous genes upon induction of individual DSBs and at different, non-repetitive, genomic loci.

To validate this observation at individual genomic sites, we selected 6 genes bearing the most frequently-cut AsiSI-sites with different position respect to the gene unit (TRIM37, RBMXL1 and HUNK cut in promoters or early TSS; GNE and MIS12; KLF7 cut in 3'UTR) and we tested the impact of DICER and DROSHA inactivation on their DISC. By RT-qPCR, we observed that DICER and DROSHA co-KD (Figure S2 A) significantly sustained transcription of all 6 DSB-bearing genes (Figure 2 B). In this setting, as additional control we confirmed that the individual KD of DICER and DROSHA KD is sufficient to diminish DISC (Figure S2B and C).

We previously reported that DROSHA and DICER are required for ATM activation [6, $15,18]$. Thus, to confirm an impact of DROSHA and DICER co-KD on ATM activation in this system, we stained cells for phosphorylated, and thus activated, ATM (pS1981pATM). pATM foci formation was quantified in all the samples in parallel by automatic Cell-Profiler software analyses (see material and methods and Figure S2D). We confirmed that DROSHA and DICER inactivation verified by western blotting (Figure $\mathrm{S} 2 \mathrm{E}$ ), reduces pATM foci formation while leaving $\gamma \mathrm{H} 2 \mathrm{AX}$ signals unaltered (Figure 
supplementary $2 \mathrm{~F}$ and G). These results confirm the role of DROSHA and DICER in promoting ATM activation (Figure Supplementary 2 E, F and G) as we and others previously reported $[6,15,25,37]$ and per se provides an additional mechanism by which DISC is mediated by ATM.

DNA damage can occur in different forms. To exclude the possibility that our observations are limited to DSB induced by restriction enzymes only, we decided to use laser micro-irradiation followed by EU incorporation to induce DNA damage in a more complex and not site-specific fashion and detect DICS as previously performed in [38] and [28]. We used U2OS expressing MDC1-fused to GFP to visualize the optical plane of the nucleus at the microscope and induce DNA damage accurately by laser microirradiation, as we previously reported [18]. After 72 hours of DROSHA and DICER co-KD (Figure S2 C), we micro-irradiated areas in cell nuclei. Immediately after DNA damage generation, cells were exposed to a 1-hour pulse of EU to label newlysynthetized RNA. Upon EU detection, combined with indirect IF against $\gamma \mathrm{H} 2 \mathrm{AX}$, we measured DISC. By expressing EU incorporation levels over $\gamma \mathrm{H} 2 \mathrm{AX}$ signals, we clearly detected transcriptional silencing of damaged chromatin (Figure $2 \mathrm{C}$ and D). DROSHA and DICER co-KD caused a higher EU signal in $\gamma \mathrm{H} 2 \mathrm{AX}$-positive areas compared to control siRNA (siCTRL) cells (Figure $2 \mathrm{C}$ and D) - ATM inactivation could not be used as positive control because in this setting it significantly reduced $\gamma \mathrm{H} 2 \mathrm{AX}$ signal. These results further demonstrate that also upon DNA damage generated by laser microirradiation DISC is controlled by DROSHA and DICER.

It has been reported that the antibiotic enoxacin stimulates the endoribonuclease activity of the DICER complex by facilitating the interaction between HIV TAR RNA binding protein, TRBP) and its RNA substrates \{Melo, $2011 \# 3634\}$. Recent work from our group demonstrated that enoxacin administration boosts DDR signaling and DNA damage repair by increasing DICER-dependent DDRNAs biogenesis [37]. Therefore, we tested if enoxacin could boost DISC. To do so, DIvA cells were treated with enoxacin before induction of damage and DISC was measured by RT-qPCR at the previously validated DSB-bearing genes. Intriguingly, enoxacin treatment significantly enhanced DISC at DSB-bearing genes (Figure 2E). To confirm enoxacin impact on DDR, we monitored $\gamma \mathrm{H} 2 \mathrm{AX}$ and 53BP1 foci formation in treated DIvA cells and quantified the number of foci per nucleus by Cell Profiler and, as expected [37], in cut cells treated with enoxacin the number and size of 53BP1 foci was significantly 
increased compared to cut untreated cells (Figure supplementary 2I) without any significant difference in the number of $\gamma \mathrm{H} 2 \mathrm{AX}$ foci (Figure supplementary $2 \mathrm{H}$ ). These results indicate that enoxacin, by stimulating DDRNA production and DDR signaling, also strengthens DISC of DSB-bearing genes, further confirming the functional involvement of DDRNA in this mechanism.

Altogether, these results suggest that DROSHA, DICER and DDRNA-mediated DDR signaling have a strong impact on transcriptional control of genes adjacent to DSB generated by different means.

\section{DICER and DROSHA controls BMI1 recruitment to DSB.}

PRC1-mediated mono-ubiquitylation of histone H2A on lysine 119 (H2A-K119ub) is known to induce chromatin compaction and transcriptional repression in different contexts [39] and contribute to DNA repair [30]. Previous work in the U20S 2-6-3 cellular system indicated that upon DSBs generation, DISC is enforced by ATM via the phosphorylation of the transcription elongation factor ENL, an event that stimulates the recruitment of the PRC1 component and E3-ubiquitin ligase BMI1 to the reporter gene[29]. Intriguingly, BMI1 has also been shown to physically interact with the DDR sensor NBS1 $[30,40]$ component of the MRN complex required for DDRNA precursors synthesis and functions $[6,9,15]$. These observations suggest a model in which PRC1 is actively recruited to the DSB cluster, and here ubiquitinates chromatin leading to DISC. Nevertheless, the recruitment of BMI1 at individual DSBs at endogenous genes in the DIvA cellular system has been questioned [41]. Thus, we decided to address the role of BMI1 in DISC of DSB-bearing genes in DIvA cells. We inactivated BMI1 by siRNAs in this cell system and measured the level of transcription of the set of six previously-validated genes. BMI1 KD (Figure 3E) inhibits DISC to an extent similar to that observed for ATM KD (Figure 3A). Next, to mechanistically link DISC in the DIvA cell line with PRC1-dependent histone ubiquitination, we stained BMI1-KD cells with antibodies against ubH2A-K119 and $\gamma \mathrm{H} 2 \mathrm{AX}$. Indeed, BMI1 KD significantly reduced the formation of detectable $\mathrm{ubH} 2 \mathrm{~A}-\mathrm{K} 119$ foci, while leaving $\gamma \mathrm{H} 2 \mathrm{AX}$ foci unaltered (Figure 3 B-D). These results support the notion that BMI1 controls chromatin ubiquitination at individual DSB and is required for DISC of DSB-bearing genes also in DIvA cells. 
Given the role of DROSHA and DICER in stimulating ATM-dependent DDR signaling $[1,6,15,17,18,20]$, we reasoned that they might also be required for DISC by modulating BMI recruitment and $\mathrm{ubH} 2 \mathrm{~A}-\mathrm{K} 119$ ubiquitination at DSB. To test this, we evaluated if the level of chromatin ubiquitination in DIvA cells was affected by DROSHA and DICER co-KD. Thus, we performed IF and chromatin immunoprecipitation (ChIP) analyses of $\mathrm{ubH} 2 \mathrm{~A}-\mathrm{K} 119$ and $\gamma \mathrm{H} 2 \mathrm{AX}$ in cells co-KD for DROSHA and DICER, or ATM as control. We observed that DROSHA and DICER inactivation reduces the number of ubH2A-K119 foci per nucleus, while leaving $\gamma \mathrm{H} 2 \mathrm{AX}$ foci number almost unaffected, (Figure 4 A, B and C). As expected, ATM KD cells also show a significant reduction in $\gamma \mathrm{H} 2 \mathrm{AX}$ signal (Figure $4 \mathrm{~A}$ and $\mathrm{B}$ ). ChIP-qPCR analyses of $\mathrm{ubH} 2 \mathrm{~A}-\mathrm{K} 119$ and $\gamma \mathrm{H} 2 \mathrm{AX}$ at three AsiSI sites (DS1, 2 and 3) revealed a strong accumulation of ubH2A-K119 signal upon DNA damage, which was, at least in part, ATM dependent (Figure $4 \mathrm{D}$ and E). An unrelated region far from AsiSI sites is used as negative control. Similarly, DROSHA and DICER co-KD (Figure S3 A) significantly reduced the accumulation of ubH2A-K119 on damaged chromatin (Figure 4E). Next, to test if the recruitment of BMI1 is controlled by DROSHA and DICER, we performed a ChIP-qPCR analysis for BMI1 at the same three DSBs in control or DROSHA and DICER co-KD cells (Figure S3 B). We observed a strong recruitment of BMI1 at both DSB in transcribed genes which was lost upon DROSHA and DICER co-KD (Figure 4F). Moreover, ATM KD led to a comparable loss in BMI1 recruitment at DSB compared to DROSHA and DICER co-KD, further strengthening the relevance of these two factors in DISC.

Finally, to support the evidence that DROSHA and DICER control the recruitment of BMI1 at DNA ends, we employed DNA Damage In Situ Ligation Followed by Proximity Ligation Assay (DI-PLA) [42], a technique that labels DNA ends through their ligation to a biotinylated DNA oligonucleotide that is detected by PLA with an anti-biotin antibody in combination with anti-BMI1 antibody. Indeed, DI-PLA signals for BMI1 increased upon DNA damage generation, again confirming that BMI1 is recruited to DSBs. Consistently with the result obtained by ChIP-qPCR, DROSHA and DICER co-KD (Figure S3 C) completely abolished BMI1 DI-PLA signal in damaged cells (Figure $4 \mathrm{G}$ and $\mathrm{H}$ ). Importantly, BMI KD completely abolished DI-PLA signal confirming the specificity of the signal (Figure S3 D). Finally, we confirmed by western 
blotting that BMI1 protein level is unchanged upon DROSHA and DICER co-KD cells, thus excluding indirect effects (Figure S3 E).

These results demonstrate that DROSHA and DICER are required for BMI1 recruitment to DSB at actively-transcribed genes.

\section{BMI1 binds to DDRNAs and antisense oligonucleotides against DDRNAs prevent}

DISC.

PRC1 complex and BMI1 has been previously shown to be recruited to their site of action via the interaction with ncRNA[43]. Thus, to probe a mechanistic link between DROSHA, DICER and BMI1 recruitment to DSBs we tested whether BMI1 directly associates with DDRNAs. To this end, we performed RNA immuno-precipitation (RIP) assays against endogenous Bmil in a cell line (NIH2/4) [44] in which we previously characterized DDRNAs accumulation upon DSB induction [6, 15, 37]upon expression of the I-SceI endonuclease $[6,15,37]$. The presence of LAC-operon repeats flanking the cut site allows the visualisation of the damaged locus and DDR focus formation upon expression of a LAC-R fused to a Cherry-tag (Figure S4 A and B). DDRNA production upon ISce-I expression has been previously characterized by small RNA-seq and qRT-PCR. We observed that immunoprecipitated Bmil associates to DDRNAs generated at the damaged site and that, importantly, such interaction increased upon DSB induction (Figure 5A). miR-125a and snoRNA U61, two other small non-coding RNAs, poorly bound to Bmil and the interaction remained unaltered upon damage (Figure 5A). Percentage of cut induction upon ISce-I expression was evaluated by IF against 53BP1 e $\gamma \mathrm{H} 2 \mathrm{AX}$ (Figure S4A) and quantified as co-localization between Cherry-LAC signal and foci for these DDR markers (Figure S4 B). This result indicates that Bmil associates specifically with DDRNAs, and that this association is promoted by DSB formation suggesting that DDRNA are relevant to mediate PRC1 dependent chromatin modification at DNA damage site.

To strengthen this conclusion, we decided to use antisense oligonucleotides (ASOs) to target DDRNAs function and test if DDRNAs inhibition is sufficient to prevent DISC. To do so we took advantage of a cellular system based on a CRISPR-Cas9 driven, sitespecific DNA damage at a reporter GFP-gene locus integrated in the genome of HeLa cells. First, we confirmed that GFP transcription is repressed by CRISPR-Cas9 mediated DSB induction using a guide RNA targeting GFP gene 5' end and that DICER and 
DROSHA co-KD strongly reduces DISC also in this cell system (Figure 5B). Next, we co-KD DROSHA and DICER and we next transfected the guide RNA and Cas9. One day later, we collected cells RNA for RT-qPCR analyses of GFP expression. DROSHA and DICER KD was confirmed by RT-qPCR (Figure S4 C). Indeed, as previously observed in DIvA cells, DSB induction represses transcription down to $50 \%$ and DROSHA and DICER co-KD abolishes DISC (Figure 5B). Similar results were obtained by ATM inhibition (Figure S4 D). GFP expression in cut samples is express relative uncut cells with the same $\mathrm{KD}$, ruling out indirect effects due to microRNA loss. DICER/DROSHA co-KD do not alter GFP's transcript expression in uncut conditions, as shown by the relative GFP expression in control versus co-KD, uncut cells (Figure S4 E).

We next designed a set of locked nuclei acids (LNA) ASOs that by stably binding and inhibiting DDRNA prevent their functions, as we demonstrated in $[6,20,21]$. Importantly, ASOs sequences were designed to target DDRNAs generated up-stream and down-stream to DSB (Figure 5C). ASO targeting DDRNAs were transfected together with Cas9 and guide RNA (scrambled or GFP) and relative GFP expression was measured by RT-qPCR. Strikingly, the administration of ASOs targeting DDRNAs prevents DISC, while control ASOs do not (Figure 5 D).

This result shows that RNA generated de novo upon damage from sequences flanking the break site plays a direct role in mediating the DISC of canonical, transcriptionallyactive genes.

In summary, this set of results provide evidence that de novo ncRNA transcription not only is required for DDR signalling activation but it also controls transcriptional silencing of pre-existing transcriptionally-active genes encoded by the same locus.

\section{Discussion}

It is now well established that DNA lesions, generated by different means, cause transcriptional repression of neighboring genes. The dampening of active transcription in cis to the damage, a phenomenon recently named with the acronym DISC by others [28], is not just a passive event caused by the physical interruption of the DNA template. Instead, it is an active process that involves the recruitment of the repressive complex PRC1 to damaged chromatin in an ATM-dependent manner. Concomitantly, a de novo transcription starting from DNA ends has been now consistently observed in different 
study and different contexts [16, 45]. DDRNAs have been originally shown to be generated both in exogenously integrated repetitive locus [15] and at endogenous deprotected telomeres [20]. In addition, we published evidences showing that DDRNA's precursors dilncRNA, are generated also at DSB generated at not-repetitive, endogenous loci induced by different means among which RNA-directed CRISPR-cas9 mediated cleavage [6]. A recent report has questioned the ubiquitous biogenesis of DDRNAs at DSBs occurring at non-repetitive loci [5]. To this regard, the present study provides additional evidences that DROSHA, DICER and their small RNA product DDRNAs play an active role at individual DSBs occurring at endogenous gene's promoters.

In this work, for the first time we mechanistically connect these two apparently contradictory events showing how endogenous non coding RNA, processed by the RNA interference machinery, control chromatin state and as a consequence transcription of protein coding genes of the same locus. This is not very different to what was described in $S$. pombe for the deposition, and then restraining, of constitutive heterochromatin at centromeres [46-48]. More in general, the role of the RNA interference in the chromatinmediated transcriptional control was extensively reported [49-52].

We show that repression of canonical genes requires the local production of short DROSHA- and DICER-dependent ncRNA, DDRNA, that associate with the ubiquitin ligase BMI1 and recruit it to the damaged locus thus providing new mechanistic details of how a damaged locus mediates PRC1 recruitment, chromatin modification and DISC in a sequence specific fashion. Importantly, BMI1 overexpression is an important feature of cancer stem cell and has been implicated in proliferation, invasion, distal metastasis [53] and chemosensitivity by contributing to both NHEJ and HR [40]. Indeed, BMI-mediated transcriptional repression of active genes promotes DNA repair [30]. Other published reports support the notion that DROSHA and DICER control the recruitment at DSB of chromatin modifiers such as the Methyltransferase MMSET and the acetyltransferase Tip60 with the final effect of enhancing local levels of histone $\mathrm{H} 4$ di- and tri-methylation at lysine 20 (H4K20me2, 3) and $\mathrm{H} 4$ acetylation at lysine 16 (H4K16Ac) [12]. In this same report, it was proposed that short ncRNA-mediated chromatin remodeling promoted DSB repair by enhancing the recruitment of repair factors such as RAD51 and BRCA1 to the DSB site. Apparently, multiple mechanisms converge on ATM activation to repress canonical gene transcription of DSB-flanking genes $[54,55]$. As an example, upon DNA damage ATM and p53 have been shown to 
modulate not only transcription but also transcript stability $[54,55]$ and recently ATM has also been shown to recruit to damaged chromatin the Bromodomain Containing 7 (BRD7) protein that binds to chromatin and brings together PRC2 and NuRD complex to achieve DISC [55]. ATM also recruits the E3 ubiquitin ligase RNF168 essential for DNA repair: RNF8/RNF168- and BMI1-dependent ubiquitination of H2A and H2AX at sites of DNA damage are thought to be independent from each other [30]. Nevertheless, RNF8 was shown to promote BMI1 recruitment to DNA damage sites [56]. Thus, the connection between RNF168- and PRC1-related ubiquitination at site of DNA damage still needs to be clarified but it appears already clear that DISC and DNA repair are intimately connected. Moreover, the set threonine kinase DYRK1B, already involved in transcription, has been described as a target of ATM that orchestrate DISC by recruiting G9a methyl transferase to damaged chromatin [28]. DYRK1B is recruited to break site in a PARP1 dependent manner and contributes to maintaining genome stability at transcribed genes. Importantly, DYRK1B also controls components of the PBAF complex but do not control BMI1 recruitment suggesting the existence of multiple parallel pathways to achieve DISC, crossing at ATM activation [28].

Formation of DNA:RNA hybrids has been reported to occur at double strand breaks and to recruit specific DNA repair factors such as BRCA1, BRCA2, and RAD51. Others have proposed that DROSHA is responsible for hybrid generation at site of DNA break $[57,58]$. Indeed DNA:RNA hybrids have been also reported to form at transcription pause site to induce termination [59] and recruit BRCA1 to counteract accumulation of hybrid-driven DNA damage via the local generation of RNAi dependent small ncRNAs[13, 60]. So, apparently DNA:RNA hybrids at transcription pausing site can induce antisense de novo transcription that generates dsRNAs processed by DICER and AGO2 generating RNAi dependent sncRNAs that mediate the recruitment of both repressive chromatin modifiers and DNA repair factors $[13,60]$.

Previous studies proposed that transcriptional downregulation is achieved by exclusion of RNA polymerase II (RNAPII) from damaged chromatin [61]. Nevertheless, we observed that RNAPII elongation is reduced in the chromatin surrounding the DNA lesion while the total amount of RNAPII protein remains mostly unaltered [26]. This evidence suggests the existence of a controlled switch in RNAPII elongation. The data here proposed suggest that DDRNAs dampen transcription of a break-flanking genes by modulating chromatin ubiquitination and possibly compaction thus inducing a rapid switch from transcription elongation to pausing of RNAPII without the need of totally 
removing RNAPII from damaged chromatin. Indeed, also previous reports suggest that occupancy of total RNAPII at damaged chromatin is not affected [23] while amount of the elongating form of RNAPII (Ser2-phosphorylated) is reduced upon damage generation[23]. This observation is compatible with a model in which DNA damage and the biogenesis of DDRNA recruit PRC1 complex to converts chromatin and RNAPII machinery from an active processive state producing high levels of mRNA to a posing state that allows recruitment of downstream DDR factors.

Overall, the emerging model suggests that, as already described for X-chromosome inactivation [62], and deposition of centromeric constitutive heterochromatin [63, 64], transcriptional repression of break-flanking genes requires the synthesis of ncRNA processed by the RNAi-machinery.

\section{Methods}

\section{Cell culture}

U20S 2-6-3 cells expressing both the YFP-MS2 viral coat protein and a "tet-on" transactivator were grown in DMEM supplemented with $10 \%$ Tet-system approved FBS, L-glutamine and $1 \%$ Penicillin-Streptomycin at $37^{\circ} \mathrm{C}$ and $5 \% \mathrm{CO}_{2}$. In order to select cells bearing the reporter locus as well as the YFP-MS2 expression construct, cells were grown in $100 \mu \mathrm{g} / \mathrm{ml}$ and 400 hygromycin and $400 \mu \mathrm{g} / \mathrm{ml} \mathrm{G} 418$, respectively. To induce transcription of the reporter locus, $1 \mu \mathrm{g} / \mathrm{ml}$ doxycycline was added to complete growth medium for 3 hours before stopping the experiment.

U2OS MDC1-GFP cells (Hable et al., 2012, a kind gift from Guido Drexler) were grown in RPMI 1640, supplemented with $10 \%$ fetal bovine serum, $1 \%$ L-glutamine, $1 \%$ penicillin/streptomycin and puromycin $(0.8 \mu \mathrm{g} / \mathrm{ml})$. DIvA (AsiSI-ER-U20S) cell line (Iacovoni et al 2010) [34] was cultured in Dulbecco's modified Eagle's Medium (DMEM) supplemented with 10\% fetal bovine serum (FBS), 1\% L-glutamine, 1\% pyruvate, HEPES $25 \mathrm{mM}$ and $1 \%$ Penicillin/Streptomycin $(\mathrm{P} / \mathrm{S})$. Cells were grown at $37^{\circ} \mathrm{C}$ in $5 \% \mathrm{CO}_{2}$ and selected on a semi-regular basis with puromycin at a final concentration of $1 \mu \mathrm{g} / \mathrm{ml}$. To induce DNA damage, cells were treated with $300 \mathrm{nM} 4$ OHT (Sigma-Aldrich; H7904) for 4 hours.

DIvA cells were exposed to $50 \mu \mathrm{M}$ enoxacin in 100\% DMSO (E3764, Sigma-Aldrich) for 48 hours. 
HeLa cells were cultured in DMEM medium supplemented with $10 \%$ FBS, $1 \%$ Lglutamine, $1 \% \mathrm{P} / \mathrm{S}$ and were grown at $37^{\circ} \mathrm{C}$ in $5 \% \mathrm{CO}_{2}$.

NIH2/4 cells (gift from E. Soutoglou), a NIH3T3-derived cell line bearing the Lac-ISceI-Tet construct, were grown in DMEM (Lonza), supplemented with 10\% FBS tetracycline-tested, $1 \% \mathrm{~L}$-glutamine and hygromycin $\left(400 \mu \mathrm{g} \mathrm{ml}^{-1}\right)$. I-SceI expressing plasmid was transfected by Lipofectamine 2000, 20 hours before cell fixation or RNA extraction.

When indicated, cells were treated with enoxacin (557305-250mg, Sigma-Aldrich) dissolved in water and added to the growth medium at the final concentration of $25 \mu \mathrm{M}$ for 48 hours.

\section{RT-qPCR}

Standard and strand-specific RT-qPCR was performed as described in Michielini 2017. Briefly, total RNA was extracted using the RNEasy Mini Kit (Qiagen; 74106) according to the manufacturer's instructions. The isolated RNA was then treated with TURBO Dnase (Ambion) according to the manufacturer's instructions. $1 \mu \mathrm{g}$ of RNA was reverse-transcribed using the SuperScript ${ }^{\mathrm{TM}}$ IV First-Strand Synthesis System (Invitrogen; 18091050) by using random examers or strand-specific primers. Real-time quantitative PCR reactions were performed on a LightCycler® 480 II Sequence Detection System (Roche) by using QuantiTect SYBR® Green PCR kit (Qiagen; 204145). Real-time cycler conditions were set up according to the manufacturer's instructions: 1) $\mathrm{PCR}$ initial activation step: $95^{\circ} \mathrm{C} 15 \mathrm{~min}$; 2) 3-step cycling (Denaturation/Annealing/Extension): $95^{\circ} \mathrm{C} 15 \mathrm{sec}>60^{\circ} \mathrm{C} 20 \mathrm{sec}>72^{\circ} \mathrm{C} 30 \mathrm{sec}, 35$ cycles. For each reaction, $15 \mathrm{ng}$ of cDNA was used.

For a complete list of the primer sequences used see table 1.

\section{Indirect immunofluorescence (IF)}

Immunofluorescence for DDR marker was performed as described in Francia 2012. Briefly, cells were grown on coverslips, fixed in $4 \%$ paraformaldehyde or methanol:acetone 1:1. NIH2/4 mouse cells were fixed by $4 \%$ paraformaldehyde. Images were acquired using a wide field Olympus IX71 equipped with PlanApo 60×/1.40NA oil immersion objective, a Cool SNAP ES camera (Photometrics) and driven by MetaMorph software (Universal Imaging Corporation). For experiments in U20S 2-6-3 
reporter cells, more accurate representative images from one experiment were acquired with a confocal laser microscope confocal (Leica TCS SP2) by sequential scanning. Comparative immunofluorescence analyses were performed in parallel with identical acquisition parameters and exposure times using CellProfiler [65] for experiments in DIvA cells and Image J (NIH) software for experiments in U20S 2-6-3 rt-TA YFP-MS2 cells. At least 30 cells were screened for each condition in all experiments in both cell lines. All data for imaging analyses were plotted with the use of GraphPad Prism software.

\section{Transfections}

Cells were transfected with siRNA oligos (usually $10 \mathrm{nM}$ final concentration) by Lipofecatmine RNAiMAX (Life Technologies) according to the manufacturer's instructions. Subsequent analyses were carried out after 72 hours. See table 1 for a complete list of the of ON-TARGET plus SMARTpool siRNA oligonucleotides (Dharmacon) for human DICER, DROSHA and BMI1, as well as Non-targeting control siRNAs and siGENOME SMARTpool siRNA oligonucleotides for human ATM used.

Cells were plated into 6 multi-well plates so that they were $70-80 \%$ confluent at the day of transfection. Cells were transfected with plasmids by Lipofectamine LTX transfection reagent (Life Technologies) according to the manufacturer's instructions. The transfection mix was removed from cells $24 \mathrm{~h}$ later, when transfected cells were collected for subsequent analysis. mCherry-LacI-FokI-WT and mCherry-LacI-FokID450A expressing vectors were transfected in U20S 2-6-3 reporter cells by Lipofectamine LTX (Life Technologies) and 24h post transfection around $60 \%$ of the cells was scored positive for mCherry-LacI focus at the reporter array.

mCherry-LacI-FokI-WT and mCherry-LacI-FokI-D450A expressing vectors were a kind gift of R.A. Greenberg, Department of Cancer Biology, Abramson Family Cancer Research Institute, Philadelphia, USA.

\section{RNA-sequencing and data analysis}

For total RNA-seq in DIvA cells, total RNA was isolated using the RNeasy Kit (Qiagen), according to the manufacturer's instructions. mRNA-seq indexed library preparation was performed starting from total mRNA (Illumina, TruSeq Stranded mRNA) according to the manufacturer's instructions. Library quality and quantity was assessed on the 2100 Bioanalyzer High Sensitivity DNA kit (Agilent), quantified on 
Qubit dsDNA HS Assay, normalized and pooled to perform a multiplexed sequencing run. Clusters were generated on the Illumina flow cell and sequencing was carried out on NextSeq 550 System with paired-end 75 bp. All six conditions were sequenced as biological triplicates. The reads for RNA-seq experiments were aligned to the GRCh37/hg19 assembly human reference genome using the STAR aligner [66] using default settings with the parameter --quantMode GeneCounts in order to obtain gene counts. Differential gene expression analysis was performed using the Bioconductor package DESeq2 (Love et al., 2014) that estimates variance-mean dependence in count data from high-throughput sequencing data and tests for differential expression exploiting a negative binomial distribution-based model.

\section{Laser micro-irradiation}

Laser-induced DNA damage was performed as described in Francia S. et al. JCS 2016 [18] in U2OS MDC1-GFP using a Leica TCS SP5 point scanning confocal microscope equipped with a Leica HCX PL APO 63×/1.4NA oil immersion objective and an environmental microscope incubator (OKOLab) set to $37^{\circ} \mathrm{C}$ and $5 \% \mathrm{CO} 2$ perfusion. The Leica TCS SP5 confocal microscope was driven by Leica LAS AF software. Briefly, cells were cultured in glass-bottom dishes and pre-sensitized for $72 \mathrm{~h}$ in $10 \mu \mathrm{m}$ BrdU. Laser micro-irradiation was carried out using a $50 \mathrm{~mW} 405 \mathrm{~nm}$ diode laser with a $100 \%$ power output. At $2 \times$ digital magnification, multiple regions of interest (ROI) of the same size were selected in each nucleus and the $405 \mathrm{~nm}$ laser was used to scan the ROIs for 50 iterations (total dwell time per pixel $490 \mu \mathrm{s}$ ).

\section{5-ethynyl uridine (5-EU) incorporation}

5-ethynyl uridine (5-EU) staining was performed as described in [38]. Following DNA damage generation through laser microirradiation, 1 hour pulse of 5-EU $1 \mathrm{mM}$ was administered to the cells, allowing the labeling of active RNA transcription. Cell were then fixed in 4\% paraformaldehyde (PFA) and levels of EU incorporation were detected by Click-iT RNA imaging kit (Invitrogen) according to the manufacturer's instructions.

\section{Western Blotting}

Immunoblotting was performed ad described in Cabrini M. et al JCS 2021 [14]. Briefly, cells were lysed in Laemmli sample buffer (2\% sodium dodecyl sulphate (SDS), $5 \%$ glycerol, $1.5 \%$ Dithiothreitol (DTT), $0.01 \%$ bromophenol blue, $60 \mathrm{mM}$ Tris $\mathrm{HCl} \mathrm{pH}$ 
6.8). Protein solutions were sonicated (Diagenode) with three bursts of $15 \mathrm{sec}$ and heated for $10 \mathrm{~min}$ at $95^{\circ} \mathrm{C}$. A $6 \% \mathrm{SDS}$-polyacrylamide gel with a width of $1 \mathrm{~mm}$ was loaded with 15-20 $\mu$ l of lysate, along with $7 \mu \mathrm{l}$ of molecular weight markers (BioRad). Gels were run in Tris-Glycine electrophoresis buffer (25 mM Tris, $250 \mathrm{mM}$ glycine, $0.1 \%$ SDS) until the dye reached the bottom of the gel. For western blotting analysis proteins were transferred to a $0.2 \mu \mathrm{m}$ nitrocellulose membrane (BioRad Trans-Blot Turbo transfer pack) using the Trans-Blot Turbo Transfer System apparatus (BioRad). The transfer was performed at $25 \mathrm{~V}$ for $10 \mathrm{~min}$. Membranes were incubated with 5\% skim milk in TBS-Tween $(0.1 \%)$ buffer for $1 \mathrm{~h}$, followed by overnight incubation at $4{ }^{\circ} \mathrm{C}$ with primary antibody and three washes with TBS-Tween before $1 \mathrm{~h}$ incubation at room temperature with the specific HRP-conjugated secondary antibody. Chemiluminescence detection was done by incubation with Luminata Classico or Crescendo (Millipore). Proteins were visualized by autoradiography on ECL films (Amersham), using various exposure times and manually developed.

\section{Chromatinimmunoprecipitation (ChIP)}

ChIP was performed as described in Cabrini et al., 2021 [14]. Briefly, cells were crosslinked for $5.5 \mathrm{~min}$ at room temperature with Fixation Buffer (1\% formaldehyde; 100 mM NaCl; 1 mM EDTA; 0.5 mM EGTA; 50 mM HEPES pH 7.4). Cross-linking was quenched by addition of glycine $(125 \mathrm{mM})$. Fixed cells were rinsed twice in 1X PBS, collected by scraping and centrifuged at $2000 \mathrm{rpm}$ for $5 \mathrm{~min}$ at $4^{\circ} \mathrm{C}$. Pellets were resuspended in cold B1 Buffer (0.25\% Triton X-100; 1 mM EDTA; 0.5 mM EGTA; 10 $\mathrm{mM}$ Tris pH 8; Proteases inhibitors (Roche); Microcystin (Enzo Life Sciences)) by mixing for $10 \mathrm{~min}$ on a rotating wheel at $4^{\circ} \mathrm{C}$ and then centrifuged at $2000 \mathrm{rpm}$ for 5 min at $4^{\circ} \mathrm{C}$. The same steps were repeated with cold Buffer B2 $(200 \mathrm{mM} \mathrm{NaCl} ; 1 \mathrm{mM}$ EDTA; 0.5 mM EGTA; 10 mM Tris pH 8; Proteases inhibitors (Roche); Microcystin (Enzo Life Sciences)). Finally, pellets were re-suspended in cold Buffer B3 (TE 1X; EGTA $0.5 \mathrm{mM}$ ) in a suitable volume. Pellets were sonicated using a FocusedUltrasonicator Covaris (duty: 5.0; PIP: 140; cycles: 200; amplitude: 0; velocity: 0; dwell: 0; microTUBEs with AFA fiber). Sonicated chromatin was diluted in RIPA buffer (1\% TritonX-100; 0,1\% Na- Deoxycholate; 0,1\% SDS; $64 \mathrm{mM} \mathrm{NaCl} ; 10 \mathrm{mM}$ Tris $\mathrm{HCl} \mathrm{pH} 8.0$ ) to give a concentration of approximately $100 \mu \mathrm{g}$ in $400 \mu \mathrm{l}$ per ChIP. Samples were pre-cleared for $2 \mathrm{~h}$, rotating at $4^{\circ} \mathrm{C}$, with $20 \mu \mathrm{l}$ of magnetic beads (Dynabeads® Protein G, LifeTechnologies) per ChIP. Sample were then incubated 
overnight rotating at $4{ }^{\circ} \mathrm{C}$ with specific antibodies or no antibody (mock). The bound material was recovered by $2 \mathrm{~h}$ incubation with $20 \mu \mathrm{l}$ of magnetic beads per ChIP. Beads were then washed, rotating at $4^{\circ} \mathrm{C}$ for $10 \mathrm{~min}$, four times in RIPA buffer, once in LiCl buffer (250 mM LiCl; 0.5\% NP-40; 0.5\% Na Deoxycholate; 1 mM EDTA; 10 mM Tris$\mathrm{HCl} \mathrm{pH} 8$ ) and finally in $1 \mathrm{X}$ TE. ChIPed material was eluted by $15 \mathrm{~min}$ incubation at $65^{\circ} \mathrm{C}$ with $150 \mu \mathrm{l}$ Elution Buffer (1\% SDS; $10 \mathrm{mM}$ EDTA; $50 \mathrm{mM}$ Tris $\mathrm{HCl} \mathrm{pH}$ 8). Samples were reverse-crosslinked by incubation with proteinase $\mathrm{K}$ (Invitrogen) at $37^{\circ} \mathrm{C}$ for $5 \mathrm{~h}$ and then at $65^{\circ} \mathrm{C}$ overnight. DNA was cleaned up by QIAquick PCR purification column (Qiagen) according to the manufacturer's instructions and eluted in $30 \mathrm{ul}$ of elution buffer (EB). DS1, 2 and 3 correspond to AsiSI-63, AsiSI-507 and AsiSI 453 respectively. Unrelated region falls in chromosome 22.

\section{DNA damage in situ ligation proximity ligation assay (DI- PLA)}

DI-PLA was performed as previously described in [14, 67]. Briefly, cells were fixed and permeabilized as as described above for immunofluorescence. Coverslips were then washed twice for 5 min in 1x CutSmart buffer (NEB) and once in 1x blunting buffer (NEB). Afterwards, blunting was performed at room temperature for $60 \mathrm{~min}$ in a final

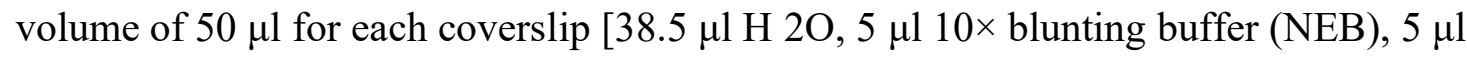
dNTP $1 \mathrm{mM}$ (NEB), $0.5 \mu \mathrm{l}$ BSA (molecular biology grade, $20 \mathrm{mg} / \mathrm{ml}$; NEB) and $1 \mu \mathrm{l}$ blunting enzyme mix (NEB)]. Coverslips were then washed twice in $1 \times$ CutSmart buffer and twice in $1 \times \mathrm{T} 4$ ligase buffer (NEB). Then, in situ ligation was performed overnight at $16^{\circ} \mathrm{C}$ in a sealed humid chamber, in $100 \mu$ final volume per coverslip using: $2 \mu 1 \mathrm{~T} 4$ Ligase $\quad$ (NEB), $\quad 5 \quad \mu l \quad 10 \mu M \quad$ biotinylated linker $\quad\left(5^{\prime}\right.$ TACTACCTCGAGAGTTACGCTAGGGA- TAACAGGGTAATATAGTTT[biotindT]TTTCTATATTACCCTGTTA- TCCCTAGCGTAACTCTCGAGGTAGTA-3'), 10 $\mu 1$ 10× T4 Ligase Buffer (NEB), $1 \mu \mathrm{d}$ dATP solution $100 \mathrm{mM}$ (NEB), $1 \mu \mathrm{l}$ BSA (molecular biology grade, $20 \mathrm{mg} / \mathrm{ml}$; NEB) and $81 \mu \mathrm{H} 2 \mathrm{O}$. Coverslips were washed twice in PBS and processed using a primary antibody against biotin partnered with a primary antibody directed against the protein under investigation (See Table 2). Following primary antibody incubation, proximity ligation assay (PLA) was performed using the Duolink In Situ Orange Starter Kit Mouse/Rabbit (Sigma-Aldrich) according to the manufacturer's protocol. Cells were washed $3 \times$ in $1 \times$ PBG and incubated with the PLA probes (secondary antibodies conjugated with oligonucleotides) for $1 \mathrm{~h}$ at $37^{\circ} \mathrm{C}$ in a humid chamber. Cells were washed twice in buffer A (supplied with the kit) and the 
ligation reaction was carried out at $37^{\circ} \mathrm{C}$ for $30 \mathrm{~min}$ in a humid chamber followed by a wash in buffer A. Cells were then incubated with the amplification mix for $1.5 \mathrm{~h}$ at $37^{\circ} \mathrm{C}$ in a darkened humidified chamber. After washing with buffer B (supplied with the kit), followed by a 1 min wash with $0.01 \times$ buffer $B$, cells were incubated with DAPI 0.2 $\mu \mathrm{g} / \mathrm{ml}$ (Sigma-Aldrich) and mounted. Images were acquired using a wide-field epifluorescence microscope (Olympus IX71) equipped with a 60x objective. Quantification of nuclear PLA dots was performed using the automated image analysis software CellProfiler 2.1.1 (Carpenter et al., 2006).

\section{RNA immunoprecipitation (RIP)}

NIH2/4 cells were transduced with a lentiviral I-SceI-GR expressing construct or with an empty vector as a control (Michelini, 2017 NCB). I-SceI-GR was translocated to the nucleus by treating cells with $0.1 \mu \mathrm{M}$ triamcinolone acetonide (T6501, Merck) for $1.5 \mathrm{~h}$ before harvesting. Cells were lysed and RIP performed as described before (Michelini, 2017 NCB). Immunoprecipitation was conducted overnight with ten micrograms of anti-BMI1 (A301-694A, Bethyl) or anti-GFP (ab290, Abcam) as a mock control. Purified RNA was retrotranscribed using miScript RT II Kit (Qiagen) and analysed by qPCR with QuantiTect SYBR Green PCR Kit (Qiagen).

\section{CRISPR-Cas9 mediated DNA cleavage and ASOs transfection in the HeLa GFP} system

HeLa cells (ATCC) were infected with a 1:1000 dilution of a lentivirus containing the pLenti-CMV-MCS-SV-puro plasmid (Addgene). Following the viral infection, cells were cultured for at least 10 passages under puromycin $(\mathrm{c}=1 \mu \mathrm{g} / \mathrm{mL})$ selection. 48 hours following siRNAs transfection, cells were transfected with Cas9 and the guide RNA (3'- GGGCACGGGCAGCTTGCCGG-5') or scrambled gRNA (sgRNA scramble FW: 3'-CACCGGCGCGAAGCTTAGGGATAAC-5'; RV: 3'AAACGTTATCCCTAAGCTTCGCGCC-5') using Lipofectamine 2000 according to the manufacturer's instructions. After 6 hours, the media was replaced by DMEM and DMSO or KUDOS (ATM inhibitor, KU60019 (Sigma-Aldrich) were added to the medium at a final concentration of $10 \mu \mathrm{M}$. DNA damage was induced by addition of 
$300 \mathrm{nM}$ 4-OHT (Sigma-Aldrich; H7904) for 4 hours. The following day cells were collected for RT-qPCR.

When cells were also transfected with ASOs (50nM), antisense oligonucleotides were transfected together with the Cas9 and the guide RNA.

For this study the following ASOs were used: 5'-AGCAAGGGCGAGGAGCTGTT-3', 5'- ACCGGGGTGGTGCCCA-3'， 5'- GAGCTGGACGGCGACGTAAA-3'， 5'TTCAGCGTGTCCGGCGAGGG-3'， 5' - TAATACCTACGGCAAGCTGA-3'， 5' TCGTGACCACCCTGACCTA-3', 5' - TGCTTCAGCCGCTACCCCGA-3'.

\section{Statistical analysis}

Fluorescence intensity results are shown as means \pm standard error of the mean (s.e.m.). qRT-PCR results are shown as means of a technical triplicate \pm standard error of the mean (s.e.m), or as mean between more than three experiments \pm standard error (s.e.m). Graphs were created and statistical analyses performed using Prism software (GraphPad). For comparative analyses in which one data series was normalized to 1, a one-sample t-test was performed on the other sample with a hypothetical mean set to 1 . For all other comparisons, a multiple unpaired two-tailed t-test was performed without assuming equal variances. For comparative analyses where number of DDR foci was investigated, a non-parametric one-way ANOVA test (because data distribution was negative using Shapiro-Wilk normality test) was applied.

\section{Antibodies}

For a complete list of the antibodies and their concentrations used in this study see table 2.

\section{Tables}

Table 1: primers used in this study.

\begin{tabular}{|l|l|}
\hline Gene & \multicolumn{1}{|c|}{ Sequence (5'-3') } \\
\hline $\begin{array}{l}\text { GAPDH_FW (housekeeping for U20S } \\
2-6-3)\end{array}$ & CTC AAG ATC ATC AGC AAT GCC \\
\hline GAPDH_REV (housekeeping) & CAT CAC GCC ACA GTT TCC C \\
\hline ECFP_FW & GAC GTA AAC GGC CAC AAG TT \\
\hline ECFP_REV & GAA CTT CAG GGT CAG CTT GC \\
\hline
\end{tabular}




\begin{tabular}{|c|c|}
\hline $\begin{array}{l}\text { B2M_FW (housekeeping for DIvA and } \\
\text { U20S-2-6-3) }\end{array}$ & TTC TGG CCT GGA GGC TAT C \\
\hline $\begin{array}{l}\text { B2M_REV (housekeeping for DIvA and } \\
\text { U20S-2-6-3) }\end{array}$ & TCA GGA AAT TTG ACT TTC CAT TC \\
\hline GNE_FW & $\begin{array}{l}\text { TTG TTA CAC TTA TCA CAG GCA } \\
\text { CAG }\end{array}$ \\
\hline GNE_REV & GAG CTT CCG TGG ATC AAT TC \\
\hline TRIM37_FW & CAG CTG CCC CTG AAG AAG \\
\hline TRIM37_REV & CGC CCA CAC TGG TAT GCT \\
\hline RBMXL1_FW & TCA GGA CTA GTT CGC AGC AG \\
\hline RBMXL1_REV & TCG AGG TGG ACC TCC ATA AC \\
\hline KLF7_FW & GGT GCA AAG CCC TTC AAA T \\
\hline KLF7_REV & CTT CAT GGA GGG CAA GAT \\
\hline HUNK_FW & CCT ACG CTG CAC CTG AAC T \\
\hline HUNK_REV & CAT GGC ATA CAT GTT CAC ACC T \\
\hline MIS12_FW & GAG AGA AGA TGA GGC GGT AGA \\
\hline MIS12_REV & GCC AAT GTC CTC AAT TTG CT \\
\hline DICER_FW & $\begin{array}{l}\text { TTA ACC TTT TGG TGT TTG ATG AGT } \\
\text { GT }\end{array}$ \\
\hline DICER_REV & GCG AGG ACA TGA TGG ACA ATT \\
\hline DROSHA_FW & GGC CCG AGA GCC TTT TAT AG \\
\hline DROSHA_REV & TGC ACA CGT CTA ACT CTT CCA C \\
\hline ATM_FW & $\begin{array}{l}\text { GCA ACT GTA CCA AGA AGA GTC } \\
\text { CAA }\end{array}$ \\
\hline ATM_REV & GCA GGA AAA AGC CAG ATG CA \\
\hline GFP_UP_Proximal_V2_FW & GACGTAAACGGCCACAAGTT \\
\hline GFP_UP_Proximal_V2_RV & GTGCAGATGAACTTCAGGGTC \\
\hline Unrelated region_FW & CCCATCTCAACCTCCACACT \\
\hline Unrelated region_REV & CTTGTCCAGATTCGCTGTGA \\
\hline DSB1_FW & GATTGGCTATGGGTGTGGAC \\
\hline DSB1_REV & CATCCTTGCAAACCAGTCCT \\
\hline DSB2_FW & CCCTGGAGGTAGGTCTGGT \\
\hline DSB2_REV & CGCACACTCACTGGTTCCT \\
\hline
\end{tabular}




\begin{tabular}{|l|l|}
\hline DSB3_FW & GGCGTACTGGTGGAAAGC \\
\hline DSB3_REV & TCAGAGTCCGAATACTTAACTACGG \\
\hline DDRNA detection & $\begin{array}{l}\text { TCCACATGTGGCCACAAATTG } \\
\text { ATCCGCTCACAATTCCACATGT } \\
\text { ATGTGGAATTGTGAGCGGAT }\end{array}$ \\
\hline hsa-miR-125a-5p & TCCCTGAGACCCTTTAACCTGTGA \\
\hline snoRNA U61 & MS00033705 (Qiagen) \\
\hline gRNA GFP & GGGCACGGGCAGCTTGCCGG \\
\hline scrambled RNAs & $\begin{array}{l}\text { CACCGGCGCGAAGCTTAGGGATAAC } \\
\text { AAACGTTATCCCTAAGCTTCGCGCC }\end{array}$ \\
\hline
\end{tabular}

Table 2: antibodies used in this study.

\begin{tabular}{|c|c|c|c|c|c|}
\hline Antibody & $\begin{array}{l}\text { Host } \\
\text { species }\end{array}$ & $\begin{array}{l}\text { Concentratio } \\
\text { n }\end{array}$ & $\begin{array}{l}\text { Applicatio } \\
\text { n }\end{array}$ & Supplier & Cat. N. \\
\hline $\begin{array}{l}\text { Anti- } \\
\gamma \mathrm{H} 2 \mathrm{AX}\end{array}$ & mouse & $1: 1000$ & IF & Millipore & $05-636$ \\
\hline $\begin{array}{l}\text { Anti- } \\
\gamma \mathrm{H} 2 \mathrm{AX}\end{array}$ & rabbit & $1: 1000$ & IF & $\begin{array}{l}\text { Cell } \\
\text { Signaling }\end{array}$ & $20 \mathrm{E} 3$ \\
\hline $\begin{array}{l}\text { Anti-ATM } \\
\text { pS1981 }\end{array}$ & mouse & $1: 400$ & IF & Rockland & $\begin{array}{l}200-301- \\
400\end{array}$ \\
\hline Anti-53BP1 & goat & $1: 2000$ & IF & Bethyl & $\begin{array}{l}\text { A303- } \\
906 a\end{array}$ \\
\hline Anti-uH2A & mouse & $1: 500$ & IF & Millipore & $05-678$ \\
\hline Anti-ATM & $\begin{array}{l}\text { Mouse } \\
\text { clone } \\
\text { SYR6D4 }\end{array}$ & $1: 1000$ & WB & $\begin{array}{l}\text { Sigma- } \\
\text { Aldrich }\end{array}$ & A6093 \\
\hline Anti-BMI1 & rabbit & $1: 2000$ & WB & Bethyl & $\begin{array}{l}\text { A301- } \\
694 A\end{array}$ \\
\hline $\begin{array}{l}\text { Anti- } \\
\text { vinculin }\end{array}$ & mouse & $1: 1000$ & WB & Millipore & $\begin{array}{l}\text { MAB357 } \\
4\end{array}$ \\
\hline $\begin{array}{l}\text { Anti- } \beta- \\
\text { tubulin }\end{array}$ & mouse & $1: 2000$ & WB & $\begin{array}{l}\text { Sigma- } \\
\text { Aldrich }\end{array}$ & T5168 \\
\hline
\end{tabular}




\begin{tabular}{|c|c|c|c|c|c|}
\hline Anti-actin & mouse & $1: 5000$ & WB & $\begin{array}{l}\text { Sigma- } \\
\text { Aldrich }\end{array}$ & A5441 \\
\hline $\begin{array}{l}\text { Anti-mouse } \\
\text { Alexa } 568 \\
\text { IgG } \\
\text { (secondary } \\
\mathrm{Ab} \text { ) }\end{array}$ & donkey & $1: 400$ & IF & $\begin{array}{l}\text { Life } \\
\text { Technologie } \\
\text { s }\end{array}$ & A10037 \\
\hline $\begin{array}{l}\text { Anti-rabbit } \\
\text { Alexa } 568 \\
\mathrm{IgG} \\
\text { (secondary } \\
\mathrm{Ab} \text { ) }\end{array}$ & donkey & $1: 400$ & IF & $\begin{array}{l}\text { Life } \\
\text { Technologie } \\
\text { s }\end{array}$ & A10042 \\
\hline $\begin{array}{l}\text { Anti-goat } \\
\text { Alexa } 488 \\
\mathrm{IgG} \\
\text { (secondary } \\
\mathrm{Ab} \text { ) }\end{array}$ & donkey & $1: 400$ & IF & $\begin{array}{l}\text { Life } \\
\text { Technologie } \\
\text { s }\end{array}$ & $\begin{array}{l}\text { A32814T } \\
\text { R }\end{array}$ \\
\hline $\begin{array}{l}\text { anti-mouse } \\
\text { Alexa } 647 \\
\mathrm{IgG} \\
\text { (secondary } \\
\mathrm{Ab} \text { ) }\end{array}$ & donkey & $1: 400$ & IF & $\begin{array}{l}\text { Life } \\
\text { Technologie } \\
\text { s }\end{array}$ & A-31571 \\
\hline $\begin{array}{l}\text { anti-mouse } \\
\text { Alexa } 647 \\
\text { IgM } \\
\text { (secondary } \\
\text { Ab) }\end{array}$ & goat & $1: 400$ & IF & Abcam & Ab150123 \\
\hline $\begin{array}{l}\text { anti- } \\
\text { UbH2AK11 } \\
9\end{array}$ & mouse & $10 \mathrm{uL}$ & ChIP & Millipore & 05-678 \\
\hline anti-BMI1 & rabbit & $1: 50$ & ChIP & $\begin{array}{l}\text { Cell } \\
\text { Signaling }\end{array}$ & 6964 \\
\hline
\end{tabular}




\begin{tabular}{|l|l|l|l|l|l|}
\hline anti-biotin & mouse & $1: 4000$ & DI-PLA & $\begin{array}{l}\text { Sigma- } \\
\text { Aldrich }\end{array}$ & B7653 \\
\hline anti-BMI1 & $\begin{array}{l}\text { Rabbit, } \\
\text { monoclona } \\
1\end{array}$ & $1: 4000$ & DI-PLA & Bethyl & BLR119H \\
& & & & \\
\hline
\end{tabular}

Table 3: siRNAs used in this study.

\begin{tabular}{|l|l|}
\hline Target mRNA & Sequence (5' '-3') \\
\hline \multirow{5}{*}{ DICER } & UAAAGUAGCUGGAAUGAUG \\
\cline { 2 - 2 } & GAAUAUGGUUGUUUGAAGA \\
\cline { 2 - 2 } & ACACAGCAGUUGUCUUAAA \\
\cline { 2 - 2 } & GAAUAUCGAUCCUAUGUUC \\
\hline DROSHA & CAACAUAGACUACACGAUU \\
\cline { 2 - 2 } & CCAACUCCCUCGAGGAUUA \\
\cline { 2 - 2 } & GGCCAACUGUUAUAGAAUA \\
\cline { 2 - 2 } & GAGUAGGCUUCGUGACUUA \\
\hline BMI1 & GAAAUUCAACCAACGGAAA \\
\cline { 2 - 2 } & ACAUAAGCAUUGGGCCAUA \\
\cline { 2 - 2 } & GGUUCGACCUUUGCAGAUA \\
\cline { 2 - 2 } & CCUCGAAAAUCAUCAGUAA \\
\hline ATM & GCAAAGCCCUAGUAACAUA \\
\hline
\end{tabular}

\section{Figure Legends}

Figure 1: DICER, similarly to ATM, appears to be required for DISC in U20S 2-

6-3 cells. A. Top: Schematic representation of the reporter locus in U20S 2-6-3 reporter cells. Bottom: qRT-PCR analysis of reporter CFP mRNA levels in uncut cells and cut cells treated with non-targeting control siRNAs (siCTRL) or siRNAs specific for DICER, DROSHA or ATM. Error bars represent SEM from 8 independent experiments. Data are depicted relative to uncut cells for each knockdown condition. $\mathrm{P}<0.01$ (**) or

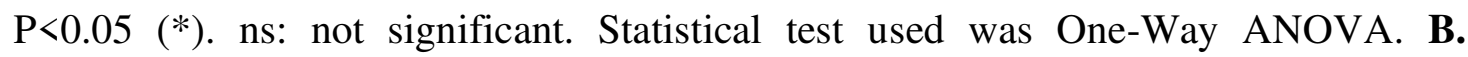
Representative images of YFP-MS2 and $\gamma \mathrm{H} 2 \mathrm{AX}$ accumulation at the Cherry-Lac locus 
in uncut, siCTRL, siDICER and siATM transfected cut cells. Scale bars: $10 \mu \mathrm{m}$ C. Quantification of YFP-MS2 fluorescent intensity from the same experimental conditions as in B. Data are depicted relative to uncut cells for each knockdown condition. Error bars represent SEM from 4 independent experiments. $\mathrm{P}<0.05$ (*) or $\left.\mathrm{P}<0.01{ }^{* *}\right)$. Statistical test used was unpaired t-test. D. Quantification of $\gamma \mathrm{H} 2 \mathrm{AX}$ fluorescence intensity from the same experimental conditions as in B. Data are depicted relative to uncut cells for each knockdown condition. Error bars represent SEM from 4 independent experiments.

Figure 2: At non-repetitive endogenous sites, DISC of break-bearing genes is controlled by DICER and DROSHA. A. Boxplot representing $\log 2$ of the fold change of transcript levels -in cells treated with non-targeting control siRNAs (CTRL), DICER and DROSHA siRNAs (siDICER,siDROSHA) or ATM siRNA (ATM)- for the 63 genes bearing cut AsiSI sites within or adjacent $( \pm 2 \mathrm{~Kb})$ to gene bodies [26] and that underwent downregulation in siCTRL upon cut. B. qRT-PCR analysis of break bearing genes expression in cut versus uncut cells treated with non-targeting, control siRNAs (siCTRL) or co-KD for DICER and DROSHA or ATM. Error bars represent SEM of fold change in expression of 18 transcripts from three independent experiments. Data are depicted relative to uncut cells for each knockdown condition. $\mathrm{P}<0.001$ (***) and $\mathrm{P}<0.0001(* * * *)$. Statistical analyses were performed by One-Way ANOVA. C. Representative images of $\gamma \mathrm{H} 2 \mathrm{AX}$ foci and EU incorporation in laser micro-irradiated DIvA cells treated with non-targeting control siRNAs (siCTRL) or siRNAs specific for DICER and DROSHA. DNA was stained by DAPI. Scale bar: $10 \mu \mathrm{m}$. D. Quantification of EU intensity in laser micro-irradiated cells treated with non-targeting control siRNAs or siRNAs specific for DICER and DROSHA. Error bars represent SEM of signal intensity of more than 200 cells from 3 independent experiments. $\mathrm{P}<0.0001$. E. qRTPCR analysis of the mRNA levels of 6 genes bearing three different AsiSI sites relative to uncut, upon mock treatment (grey bars) or with enoxacin (red bar). Error bars represent SEM of expression level of 6 genes. $\mathrm{P}<0.01$.

Figure 3: BMI1 controls DISC and histone ubH2A-K119 in DIvA cells. A. qRTPCR analysis of the mRNA levels of six different break bearing genes upon treatment with siRNAs against BMI1 or ATM or non-targeting control siRNAs (siCTRL). Data are depicted relative to uncut cells for each knockdown condition. Transcript level of 
each gene in uncut condition has been set to one. Error bars represent SEM of fold change of 18 transcripts from 3 independent experiments. $\mathrm{P}<0.0001$ (****). Statistical test used was One-Way ANOVA. B. Representative images of $\gamma \mathrm{H} 2 \mathrm{AX}$ and $\mathrm{ubH} 2 \mathrm{~A}$ K119 foci in cut and uncut cells treated with siRNAs against BMI1 or ATM or nontargeting control siRNAs (siCTRL). DNA was stained by DAPI. Scale bars: $20 \mu \mathrm{m}$. C. Quantification of $\gamma \mathrm{H} 2 \mathrm{AX}$ foci in cut and uncut cells treated with non-targeting control siRNAs or siRNAs specific for BMI1. Error bars represent SEM from more than 150 cells from two independent experiments. D. Quantification of ubH2A-K119 foci in cut and uncut cells treated with non-targeting control siRNAs or siRNAs specific for BMI1. Error bars represent SEM from more than 150 cells from two independent experiments. $\mathrm{P}<0.0001$. Statistical test used was One-Way ANOVA E. Western Blot analysis of BMI1 protein levels in uncut and cut cells treated with non-targeting control siRNAs or siRNAs against BMI1. Actin immunoblot was used as loading control.

Figure 4: DICER and DROSHA inactivation also reduces ubH2A-K119 foci and BMI1 recruitment at DNA damage sites. A. Representative images of $\gamma \mathrm{H} 2 \mathrm{AX}$ and ubH2A-K119 foci in cut and uncut cells treated with non-targeting control siRNAs (siCTRL) or siRNAs against DICER and DROSHA or ATM. DNA was stained by DAPI. Scale bars: $20 \mu \mathrm{m}$. B. Quantification of $\gamma \mathrm{H} 2 \mathrm{AX}$ foci in cut and uncut cells treated as in A. Error bars represent SEM from more than 100 cells from two independent experiments. $\mathrm{P}<0.0001$. C. Quantification of $\mathrm{ubH} 2 \mathrm{~A}-\mathrm{K} 119$ foci in cut and uncut cells treated as in A. Error bars represent SEM from more than 100 cells from two independent experiments. $\mathrm{P}<0.001(* * *)$ or $\mathrm{P}<0.0001(* * * *)$. D-F. ChIP-qPCR analysis at three AsiSI cut sites and an unrelated region in uncut and cut cells treated with nontargeting control siRNAs or siRNAs for DICER and DROSHA or ATM for the markers $\gamma \mathrm{H} 2 \mathrm{AX}(\mathrm{D}), \mathrm{ubH} 2 \mathrm{~A}-\mathrm{K} 119$ (E) and BMI1 protein (F). Error bars represent SEM from 3 independent experiments. $\mathrm{P}<0.0001 \quad(* * * *), \quad \mathrm{P}<0.01 \quad(* *)$ or $\mathrm{P}<0.05 \quad(*)$. $\mathbf{G}$. Representative images of DI-PLA signals for BMI1 recruitment to sites of damage in cut and uncut cells treated with non-targeting control siRNAs or siRNA specific for DICER and DROSHA or ATM. H. Quantification of DI-PLA signals for BMI1 recruitment in cut) and uncut cells treated with non-targeting control siRNAs or siRNAs specific for DICER and DROSHA or ATM. Error bars represent SEM from more than 100 cells from three independent experiments. $\mathrm{P}<0.05(*)$ or $\mathrm{P}<0.01(* *)$. Statistical test used was One-Way ANOVA. 
Figure 5: At sites of damage BMI1 interacts with DDRNAs, and transfecting ASOs inhibiting DDRNA is sufficient to abolish DISC. A. RNA immunoprecipitation (RIP) analysis of BMI1 followed by miScript RT II retro-transcription and qPCR analysis to detect association with DDRNAs (or miR-125a as control) in NIH2/4 cut and uncut cells. Error bars represent SEM from 3 independent experiments. $\mathrm{P}<0.05$. Statistical test used was Two-Way ANOVA. B. qRT-PCR analysis of GFP mRNA levels in cut cells relative to uncut ones set to one in HeLa cells with an integrated GFP gene (HeLa-GFP) treated with control siRNAs siCTRL or siRNAs against DROSHA and DICER. Error bars represent SEM from 3 independent experiments. $\mathrm{P}<0.05$. Statistical test used was paired Student t-test. C Schematic representation of the construct integrated in HeLaGFP used to evaluate gene expression following CRISPR-Cas9 cleavage. ASOs: sequence-specific antisense oligonucleotides. D. qRT-PCR analysis of GFP mRNA levels in cut and uncut cells treated with non-targeting control ASOs or ASOs specific for the dilncRNAs generated at this locus. Error bars represent SEM from 3 independent experiments. Data are depicted relative to uncut cells. $\mathrm{P}<0.01$. Statistical test used was paired Student t-test.

Figure Supplementary 1: One representative qRT-PCR analysis of ATM mRNA levels in both cut and uncut U20S 2-6-3 cells treated with non-targeting control siRNAs (black) or siRNAs specific for ATM (grey). B. One representative qRT-PCR analysis of DICER mRNA levels in both cut and uncut U20S 2-6-3 cells treated with nontargeting control siRNAs (black) or siRNAs specific for DICER (grey). C. One representative qRT-PCR analysis of DROSHA mRNA levels in both cut and uncut U20S 2-6-3 cells treated with non-targeting control siRNAs (black) or siRNAs specific for DROSHA (grey).

Figure supplementary 2: A. One representative qRT-PCR analysis of DICER, DROSHA or ATM mRNA levels in both cut and uncut DIvA cells treated with nontargeting control siRNAs (black) or siRNAs specific for DICER, DROSHA or ATM (grey). B. qRT-PCR analysis of break bearing genes expression in cut versus uncut cells treated with non-targeting control siRNAs (siCTRL) or siRNAs specific for DICER, DROSHA or ATM. Error bars represent SEM of fold change in expression of 18 transcripts coming from three independent experiments. Data are depicted relative to 
uncut cells for each knockdown condition. $\mathrm{P}<0.001$ (***) or $\mathrm{P}<0.0001$ (****). Statistical analyses were performed by One-Way ANOVA C. One representative qRTPCR analysis of DICER, DROSHA or ATM mRNA levels in both cut and uncut DIvA cells treated with non-targeting control siRNAs (black) or siRNAs specific for DICER, DROSHA or ATM (grey). D. Representative images of $\gamma \mathrm{H} 2 \mathrm{AX}$ and pATM foci in cut and uncut DIvA cells treated with a non-targeting control siRNA or siRNAs specific for DICER and DROSHA or ATM. Scale bar: $20 \mu \mathrm{m}$. E. Western Blot analysis of DICER and DROSHA protein levels in cells treated with non-targeting control siRNAs or siRNAs specific for DICER and DROSHA. TUBULIN immunoblot was used as a loading control. F. Quantification of $\gamma \mathrm{H} 2 \mathrm{AX}$ foci in in cut (grey) and uncut (black) cells treated as in D. Error bars represent SEM. P<0.0001. G. Quantification of pATM foci in cut and uncut cells treated as in D. Error bars represent SEM. $\mathrm{P}<0.0001$. Statistical analyses were performed by One-Way ANOVA in F and G. H. Quantification of the number of $\gamma \mathrm{H} 2 \mathrm{AX}$ foci in cut and uncut cells treated or non-treated with enoxacin. Error bars represent SEM from more than 50 cells from one representative experiment. $\mathrm{P}<0.001$ I. Quantification of the number of 53BP1 foci in cut and uncut cells treated or non-treated with enoxacin. Error bars represent SEM from more than 50 cells from one representative experiment. $\mathrm{P}<0.0001$. Statistical analyses were performed by One-Way ANOVA.

Figure supplementary 3: A. One representative qRT-PCR analysis of DICER, DROSHA and ATM mRNA levels in cut and uncut cells treated with non-targeting control siRNAs (black) or siRNAs specific for DICER and DROSHA or ATM (grey). B. One representative qRT-PCR analysis of DICER, DROSHA and ATM mRNA levels in cut and uncut cells treated with non-targeting control siRNAs (black) or siRNAs specific for DICER and DROSHA or ATM (grey). C. One representative qRT-PCR analysis of DICER, DROSHA and ATM mRNA levels in cut and uncut cells treated with non-targeting control siRNAs (black) or siRNAs specific for DICER and DROSHA or ATM (grey). D. Quantification of DI-PLA signals for BMI1 recruitment to sites of DNA damage in cut (grey) and uncut (black) cells treated with control siRNAs or siRNAs specific for BMI1. Error bars represent SEM from 2 independent experiments. $\mathrm{P}<0.0001$. Statistical analyses were performed by One-Way ANOVA. E. Western Blot analysis of DICER, DROSHA and BMI1 protein levels in cut and uncut 
cells treated with non-targeting control siRNAs or siRNAs specific for DICER and DROSHA. VINCULIN immunoblot was used as a loading control.

Figure Supplementary 4: A. Representative images of Cherry-Lac, 53BP1 and $\gamma \mathrm{H} 2 \mathrm{AX}$ foci in uncut (top row) and cut (bottom row) cells. DNA was stained by DAPI. Scale bars: $10 \mu \mathrm{m}$. B. Percentage of co-localization analysis of Cherry-Lac and $\gamma \mathrm{H} 2 \mathrm{AX}$ or 53BP1 foci in cut and uncut cells. Cut efficiency is demonstrated by increase of colocalization C. One representative qRT-PCR analysis of DICER and DROSHA mRNA levels in cut and uncut cells treated with non-targeting control siRNAs (black) or siRNAs specific for DICER and DROSHA . D. qRT-PCR analysis of GFP mRNA levels in cut e uncut cells treated with DMSO or ATM inhibitor (ATMi). Error bars are SEM from 3 independent experiments. P<0.01. E. qRT-PCR analysis of GFP mRNA levels in uncut cells treated with non-targeting control siRNAs or siRNAs specific for DICER and DROSHA. Error bars represent SEM from 3 independent experiments. ns= not significant.

\section{Acknoledgments}

We thank Dr.Paolo Colombi for useful discussion and help in commenting on the text and all the components of the laboratory for useful discussion. The research was funded by EPIGEN, Progetto Bandiera Epigenomica; Fondazione Cariplo project 2014-1215. S.F. is supported by AriSLA (projects "DDRNA\&ALS" and its follow up "DDR\&ALS") and by a grant POR FESR 2014-2020 Regione Lombardia (InterSLA project). S.M. is supported by IUSS Pavia. F.d'A.d.F laboratory is supported by: ERC advanced grant (TELORNAGING-835103); AIRC-IG (21762); Telethon (GGP17111); AIRC 5X1000 (21091); ERC PoC grant (FIREQUENCER875139); Progetti di Ricerca di Interesse Nazionale (PRIN) 2015 "ATR and ATMmediated control of chromo- some integrity and cell plasticity"; Progetti di Ricerca di Interesse Nazionale (PRIN) 2017 "RNA and genome Instability"; Progetto AriSLA 2021 “DDR \& ALS”; POR FESR 2014-2020 Regione Lombardia (InterSLA project); FRRB-Fonda- zione Regionale per la Ricerca Biomedica- under the frame of EJP RD, the European Joint Programme on Rare Diseases with funding from the European Union's Horizon 2020 research and innovation program under the EJP RD COFUND-EJP NO 825575. 


\section{Author contribution}

SF conceived the study with the help of FdAdF and designed the experiments. SM execute RT-qPCR in DIvA cells treated with enoxacin, assembled figure legends and material and methods and edited the text. FI performed bioinformatic analyses of RNA sequencing of Figure 2A and edited the relative part in the result's section. UG performed IP for Bmi1 In NIH2/4 cellular system and wrote the relative part in the results section. IC perform all the remaining experiments.

\section{References}

1. Michelini, F., et al., From "Cellular" RNA to "Smart" RNA: Multiple Roles of RNA in Genome Stability and Beyond. Chem Rev, 2018.

2. Michalik, K.M., R. Bottcher, and K. Forstemann, A small RNA response at DNA ends in Drosophila. Nucleic acids research, 2012. 40(19): p. 9596-603.

3. Wei, W., et al., A Role for Small RNAs in DNA Double-Strand Break Repair. Cell, 2012. 149(1): p. 101-12.

4. Gao, M., et al., Ago2 facilitates Rad51 recruitment and DNA double-strand break repair by homologous recombination. Cell research, 2014.

5. Bonath, F., et al., Next-generation sequencing reveals two populations of damage-induced small RNAs at endogenous DNA double-strand breaks. Nucleic Acids Res, 2018. 46(22): p. 11869-11882.

6. Michelini, F., et al., Damage-induced IncRNAs control the DNA damage response through interaction with DDRNAs at individual double-strand breaks. Nat Cell Biol, 2017. 19(12): p. 1400-1411.

7. Burger, K., M. Schlackow, and M. Gullerova, Tyrosine kinase c-Abl couples RNA polymerase II transcription to DNA double-strand breaks. Nucleic Acids Res, 2019. 47(7): p. 3467-3484.

8. Vitor, A.C., et al., Single-molecule imaging of transcription at damaged chromatin. Sci Adv, 2019. 5(1): p. eaau1249.

9. Sharma, S., et al., MRE11-RAD50-NBS1 Complex Is Sufficient to Promote Transcription by RNA Polymerase II at Double-Strand Breaks by Melting DNA Ends. Cell Rep, 2021. 34(1): p. 108565.

10. Pessina, F., et al., Functional transcription promoters at DNA double-strand breaks mediate RNA-driven phase separation of damage-response factors. Nat Cell Biol, 2019. 21(10): p. 1286-1299.

11. Burger, K., et al., Nuclear phosphorylated Dicer processes double-stranded RNA in response to DNA damage. J Cell Biol, 2017. 216(8): p. 2373-2389. 
12. Wang, Q. and M. Goldstein, Small RNAs Recruit Chromatin-Modifying Enzymes MMSET and Tip60 to Reconfigure Damaged DNA upon DoubleStrand Break and Facilitate Repair. Cancer Res, 2016. 76(7): p. 1904-15.

13. Hatchi, E., et al., BRCA1 and RNAi factors promote repair mediated by small RNAs and PALB2-RAD52. Nature, 2021.

14. Cabrini, M., et al., DROSHA is recruited to DNA damage sites by the MRN complex to promote non-homologous end joining. J Cell Sci, 2021. 134(6).

15. Francia, S., et al., Site-specific DICER and DROSHA RNA products control the DNA-damage response. Nature, 2012. 488(7410): p. 231-5.

16. d'Adda di Fagagna, F., A direct role for small non-coding RNAs in DNA damage response. Trends in cell biology, 2014. 24(3): p. 171-8.

17. Francia, S., Non-Coding RNA: Sequence-Specific Guide for Chromatin Modification and DNA Damage Signaling. Front Genet, 2015. 6: p. 320.

18. Francia, S., et al., DICER, DROSHA and DNA damage response RNAs are necessary for the secondary recruitment of DNA damage response factors. J Cell Sci, 2016. 129(7): p. 1468-76.

19. Michelini, F., et al., RNase A treatment and reconstitution with DNA damage response RNA in living cells as a tool to study the role of non-coding RNA in the formation of DNA damage response foci. Nat Protoc, 2019. 14(5): p. 1489-1508.

20. Rossiello, F., et al., DNA damage response inhibition at dysfunctional telomeres by modulation of telomeric DNA damage response RNAs. Nat Commun, 2017. 8: p. 13980.

21. Aguado, J., et al., Inhibition of DNA damage response at telomeres improves the detrimental phenotypes of Hutchinson-Gilford Progeria Syndrome. Nat Commun, 2019. 10(1): p. 4990.

22. Lu, W.T., et al., Drosha drives the formation of DNA:RNA hybrids around DNA break sites to facilitate DNA repair. Nat Commun, 2018. 9(1): p. 532.

23. Shanbhag, N.M., et al., ATM-dependent chromatin changes silence transcription in cis to DNA double-strand breaks. Cell, 2010. 141(6): p. 97081.

24. Pankotai, T., et al., DNAPKcs-dependent arrest of RNA polymerase II transcription in the presence of DNA breaks. Nature structural \& molecular biology, 2012. 19(3): p. 276-82.

25. Kakarougkas, A., et al., Requirement for PBAF in transcriptional repression and repair at DNA breaks in actively transcribed regions of chromatin. Mol Cell, 2014. 55(5): p. 723-32.

26. Iannelli, F., et al., A damaged genome's transcriptional landscape through multilayered expression profiling around in situ-mapped DNA double-strand breaks. Nat Commun, 2017. 8: p. 15656.

27. Capozzo, I., et al., Express or repress? The transcriptional dilemma of damaged chromatin. FEBS J, 2017. 284(14): p. 2133-2147.

28. Dong, C., et al., Screen identifies DYRK1B network as mediator of transcription repression on damaged chromatin. Proc Natl Acad Sci U S A, 2020. 117(29): p. 17019-17030.

29. Ui, A., Y. Nagaura, and A. Yasui, Transcriptional elongation factor ENL phosphorylated by ATM recruits polycomb and switches off transcription for DSB repair. Mol Cell, 2015. 58(3): p. 468-82. 
30. Ismail, I.H., et al., BMI1-mediated histone ubiquitylation promotes DNA double-strand break repair. J Cell Biol, 2010. 191(1): p. 45-60.

31. Yang, Q., Q.A. Ye, and Y. Liu, Mechanism of siRNA production from repetitive DNA. Genes Dev, 2015. 29(5): p. 526-37.

32. Heinrich, S., et al., Stem-loop RNA labeling can affect nuclear and cytoplasmic mRNA processing. RNA, 2017. 23(2): p. 134-141.

33. Kakarougkas A, D.J., Jeggo PA, The PBAF chromatin remodeling complex represses transcription and promotes rapid repair at DNA double-strand breaks. Mol Cell Oncol., 2015. 2(1).

34. Iacovoni, J.S., et al., High-resolution profiling of gammaH2AX around DNA double strand breaks in the mammalian genome. The EMBO journal, 2010. 29(8): p. 1446-57.

35. Aymard, F., et al., Transcriptionally active chromatin recruits homologous recombination at DNA double-strand breaks. Nature structural \& molecular biology, 2014. 21(4): p. 366-74.

36. Asada K, C.E., Fu X, Li Z, Makowski E, Wu YC, Mito JK, Kirsch DG, Baraban J, Paroo Z. , Rescuing dicer defects via inhibition of an anti-dicing nuclease. Cell Rep., 2014. 9(4): p. 1471-81.

37. Gioia, U., et al., Pharmacological boost of DNA damage response and repair by enhanced biogenesis of DNA damage response RNAs. Sci Rep, 2019. 9(1): p. 6460.

38. Gong, F., et al., Screen identifies bromodomain protein ZMYND8 in chromatin recognition of transcription-associated DNA damage that promotes homologous recombination. Genes Dev, 2015. 29(2): p. 197-211.

39. Tamburri, S., et al., Histone H2AK119 Mono-Ubiquitination Is Essential for Polycomb-Mediated Transcriptional Repression. Mol Cell, 2020. 77(4): p. 840-856 e5.

40. Lin, X., et al., A Novel Aspect of Tumorigenesis-BMI1 Functions in Regulating DNA Damage Response. Biomolecules, 2015. 5(4): p. 3396-415.

41. Chandler, H., et al., Role of polycomb group proteins in the DNA damage response--a reassessment. PLoS One, 2014. 9(7): p. e102968.

42. Galbiati, A., C. Beausejour, and F. d'Adda di Fagagna, A novel single-cell method provides direct evidence of persistent DNA damage in senescent cells and aged mammalian tissues. Aging Cell, 2017. 16(2): p. 422-427.

43. Cerase, A. and G.G. Tartaglia, Long non-coding RNA-polycomb intimate rendezvous. Open Biol, 2020. 10(9): p. 200126.

44. Soutoglou, E., et al., Positional stability of single double-strand breaks in mammalian cells. Nat Cell Biol, 2007. 9(6): p. 675-82.

45. D'Alessandro, G. and F. d'Adda di Fagagna, Transcription and DNA Damage: Holding Hands or Crossing Swords? J Mol Biol, 2017. 429(21): p. 3215-3229.

46. Volpe, T.A., et al., Regulation of heterochromatic silencing and histone H3 lysine-9 methylation by RNAi. Science, 2002. 297(5588): p. 1833-7.

47. Buker, S.M., et al., Two different Argonaute complexes are required for siRNA generation and heterochromatin assembly in fission yeast. Nat Struct Mol Biol, 2007. 14(3): p. 200-7.

48. Keller, C., et al., Noncoding RNAs prevent spreading of a repressive histone mark. Nature structural \& molecular biology, 2013. 20(8): p. 994-1000. 
49. Cernilogar, F.M., et al., Chromatin-associated RNA interference components contribute to transcriptional regulation in Drosophila. Nature, 2011. 480(7377): p. 391-5.

50. Martienssen, R.A., Maintenance of heterochromatin by RNA interference of tandem repeats. Nat Genet, 2003. 35(3): p. 213-4.

51. Martienssen, R.A., M. Zaratiegui, and D.B. Goto, RNA interference and heterochromatin in the fission yeast Schizosaccharomyces pombe. Trends Genet, 2005. 21(8): p. 450-6.

52. Peng, J.C. and G.H. Karpen, H3K9 methylation and RNA interference regulate nucleolar organization and repeated DNA stability. Nat Cell Biol, 2007. 9(1): p. 25-35.

53. Wang, M.C., et al., BMI-1, a promising therapeutic target for human cancer. Oncol Lett, 2015. 10(2): p. 583-588.

54. Venkata Narayanan, I., et al., Transcriptional and post-transcriptional regulation of the ionizing radiation response by ATM and p53. Sci Rep, 2017. 7: p. 43598.

55. $\mathrm{Hu}, \mathrm{K}$, et al., ATM-Dependent Recruitment of BRD7 is required for Transcriptional Repression and DNA Repair at DNA Breaks Flanking Transcriptional Active Regions. Adv Sci (Weinh), 2020. 7(20): p. 2000157.

56. Ginjala, V., et al., BMI1 is recruited to DNA breaks and contributes to DNA damage-induced H2A ubiquitination and repair. Mol Cell Biol, 2011. 31(10): p. 1972-82.

57. Bader, A.S. and M. Bushell, DNA:RNA hybrids form at DNA double-strand breaks in transcriptionally active loci. Cell Death Dis, 2020. 11(4): p. 280.

58. D'Alessandro, G., et al., BRCA2 controls DNA:RNA hybrid level at DSBs by mediating RNase H2 recruitment. Nat Commun, 2018. 9(1): p. 5376.

59. Skourti-Stathaki, K., N.J. Proudfoot, and N. Gromak, Human senataxin resolves RNA/DNA hybrids formed at transcriptional pause sites to promote Xrn2-dependent termination. Mol Cell, 2011. 42(6): p. 794-805.

60. Hatchi, E., et al., BRCA1 recruitment to transcriptional pause sites is required for R-loop-driven DNA damage repair. Mol Cell, 2015. 57(4): p. 636-647.

61. Beli, P., et al., Proteomic investigations reveal a role for RNA processing factor THRAP3 in the DNA damage response. Molecular cell, 2012. 46(2): p. 21225.

62. Bousard, A., et al., The role of Xist-mediated Polycomb recruitment in the initiation of X-chromosome inactivation. EMBO Rep, 2019. 20(10): p. e48019.

63. Fukagawa, T., et al., Dicer is essential for formation of the heterochromatin structure in vertebrate cells. Nat Cell Biol, 2004. 6(8): p. 784-91.

64. Volpe, T. and R.A. Martienssen, RNA interference and heterochromatin assembly. Cold Spring Harb Perspect Biol, 2011. 3(9): p. a003731.

65. Carpenter, A.E., et al., CellProfiler: image analysis software for identifying and quantifying cell phenotypes. Genome Biol, 2006. 7(10): p. R100.

66. Dobin A, D.C., Schlesinger F, Drenkow J, Zaleski C, Jha S, Batut P, Chaisson M, Gingeras TR., STAR: ultrafast universal RNA-seq aligner. . Bioinformatics., 2013. 29(1): p. 15-21.

67. Galbiati, A. and F. d'Adda di Fagagna, DNA Damage In Situ Ligation Followed by Proximity Ligation Assay (DI-PLA). Methods Mol Biol, 2019. 1896: p. 1120. 


\section{Figures}

Figure 1

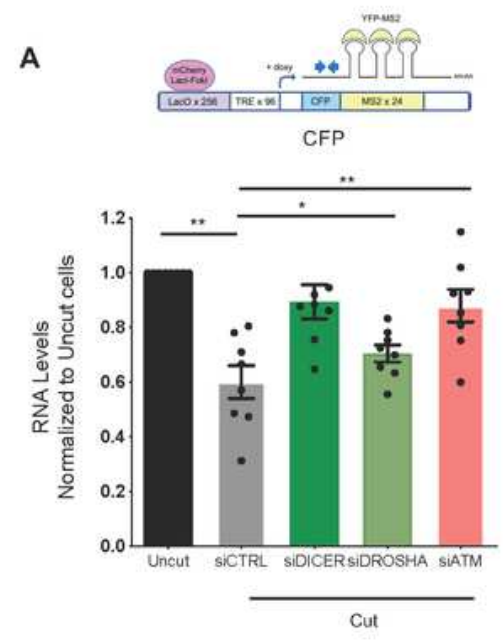

B

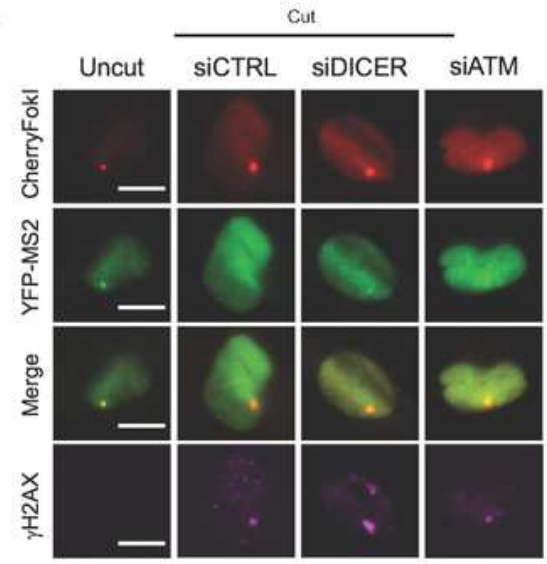

C
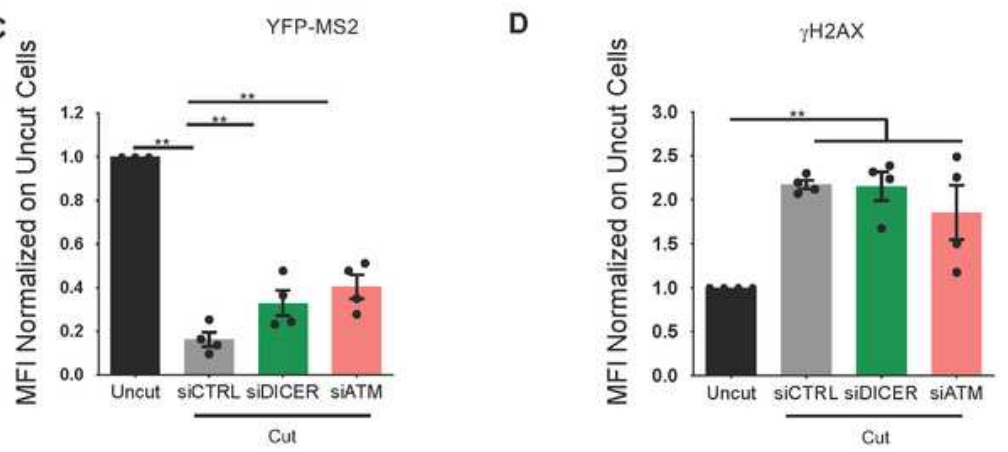

Figure 1

DICER, similarly to ATM, appears to be required for DISC in U20S 2-6-3 cells. A. Top: Schematic representation of the reporter locus in U20S 2-6-3 reporter cells. Bottom: qRT-PCR analysis of reporter CFP mRNA levels in uncut cells and cut cells treated with non-targeting control siRNAs (siCTRL) or siRNAs 
specific for DICER, DROSHA or ATM. Error bars represent SEM from 8 independent experiments. Data are depicted relative to uncut cells for each knockdown condition. $\mathrm{P}<0.01(* \star)$ or $\mathrm{P}<0.05\left({ }^{*}\right)$. ns: not significant. Statistical test used was One-Way ANOVA. B. Representative images of YFP-MS2 and YH2AX accumulation at the Cherry-Lac locus in uncut, siCTRL, siDICER and siATM transfected cut cells. Scale bars: $10 \mu \mathrm{m}$ C. Quantification of YFP-MS2 fluorescent intensity from the same experimental conditions as in B. Data are depicted relative to uncut cells for each knockdown condition. Error bars represent SEM from 4 independent experiments. $P<0.05\left(^{*}\right)$ or $P<0.01(* *)$. Statistical test used was unpaired t-test. $\mathbf{D}$. Quantification of $\mathrm{YH} 2 \mathrm{AX}$ fluorescence intensity from the same experimental conditions as in B. Data are depicted relative to uncut cells for each knockdown condition. Error bars represent SEM from 4 independent experiments. 
Figure 2.

A
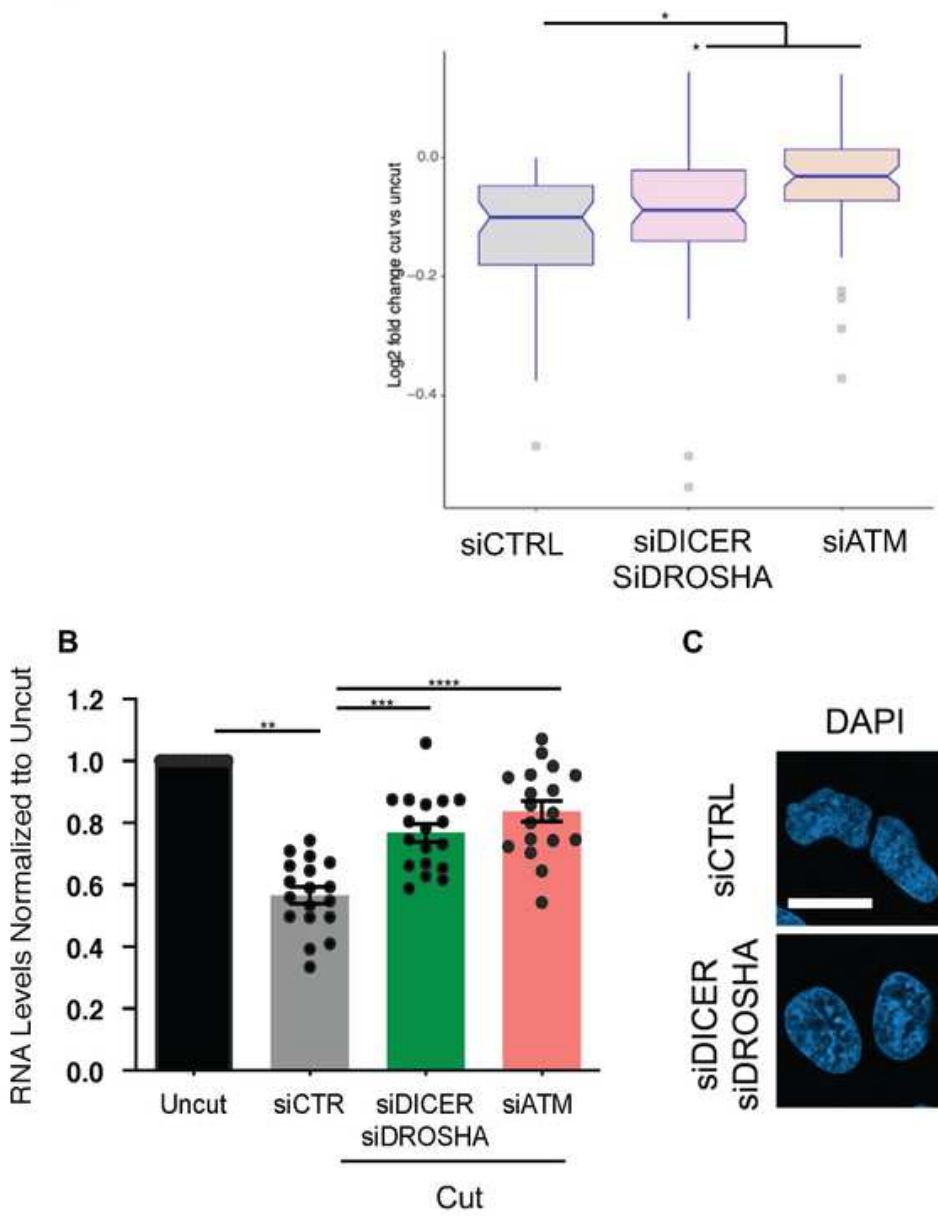

C
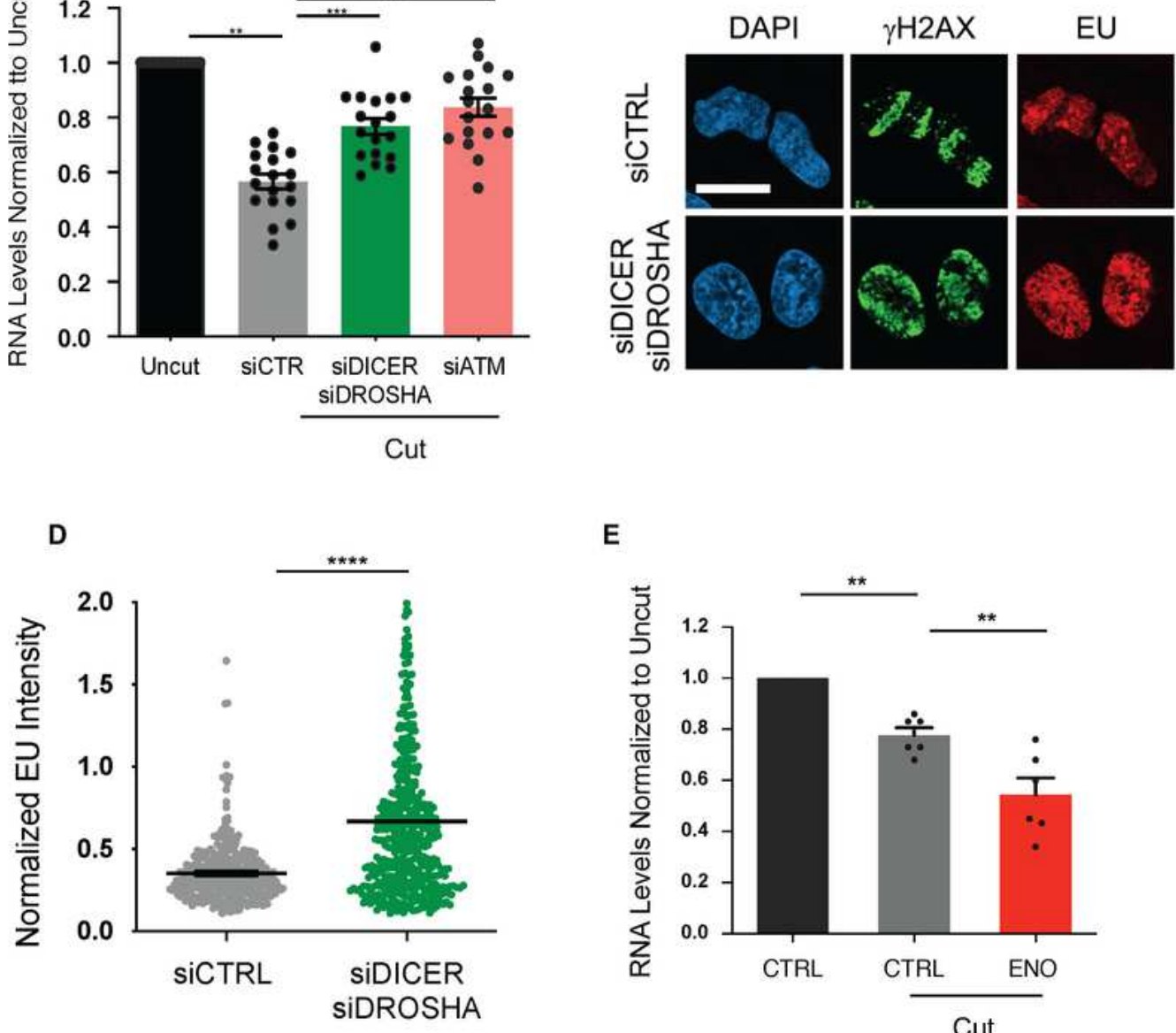

E

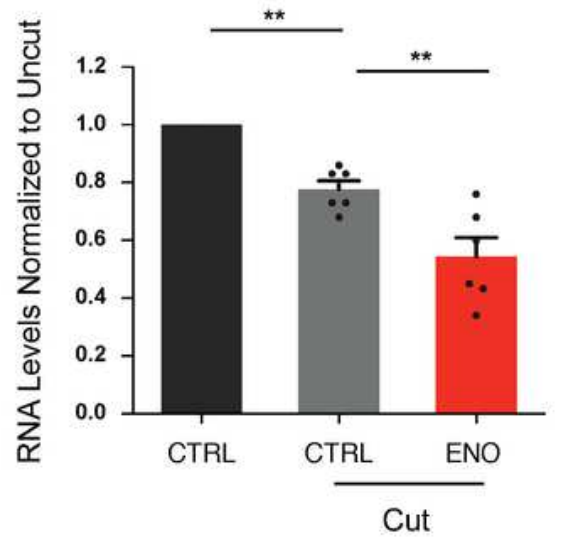

Figure 2

At non-repetitive endogenous sites, DISC of break-bearing genes is controlled by DICER and DROSHA. A. Boxplot representing log2 of the fold change of transcript levels -in cells treated with non-targeting control siRNAs (CTRL), DICER and DROSHA siRNAs (siDICER,siDROSHA) or ATM siRNA (ATM)- for the 63 genes bearing cut AsiSI sites within or adjacent $( \pm 2 \mathrm{~Kb})$ to gene bodies [26] and that underwent downregulation in siCTRL upon cut. B. qRT-PCR analysis of break bearing genes expression in cut versus uncut cells 
treated with non-targeting, control siRNAs (siCTRL) or co-KD for DICER and DROSHA or ATM. Error bars represent SEM of fold change in expression of 18 transcripts from three independent experiments. Data

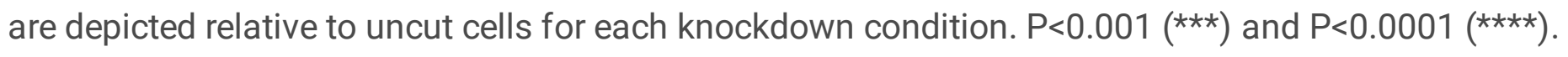
Statistical analyses were performed by One-Way ANOVA. C. Representative images of $\mathrm{YH} 2 \mathrm{AX}$ foci and EU incorporation in laser micro-irradiated DlvA cells treated with non-targeting control siRNAs (SICTRL) or siRNAs specific for DICER and DROSHA. DNA was stained by DAPI. Scale bar: $10 \mu \mathrm{m}$. D. Quantification of EU intensity in laser micro-irradiated cells treated with non-targeting control siRNAs or siRNAs specific for DICER and DROSHA. Error bars represent SEM of signal intensity of more than 200 cells from 3 independent experiments. $P<0.0001$. E. qRT-PCR analysis of the mRNA levels of 6 genes bearing three different AsiSI sites relative to uncut, upon mock treatment (grey bars) or with enoxacin (red bar). Error bars represent SEM of expression level of 6 genes. $P<0.01$. 
Figure 3

A

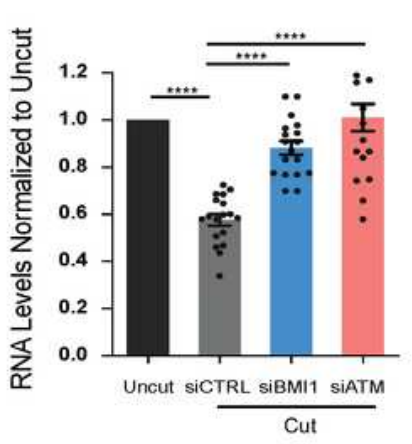

C

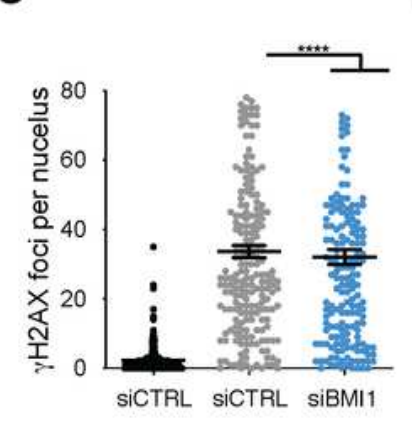

B
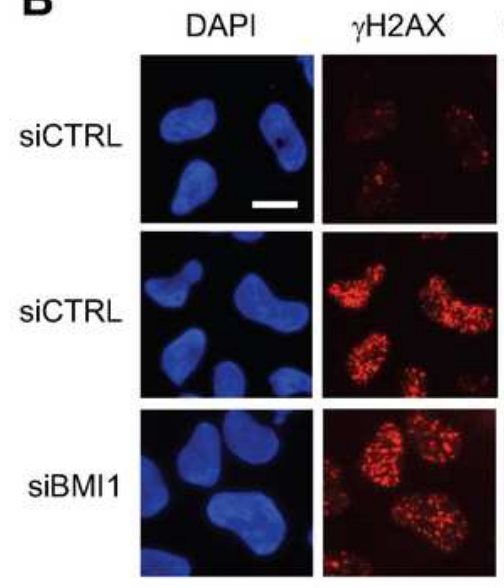

ubH2A-K119

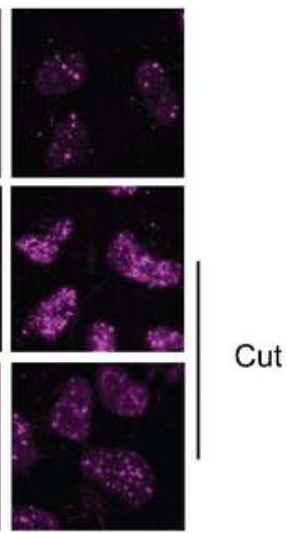

E
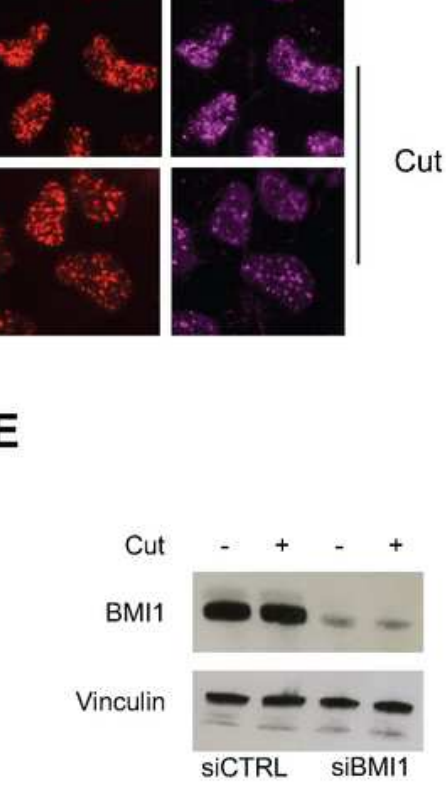

Cut

\section{Figure 3 \\ Figure 3}

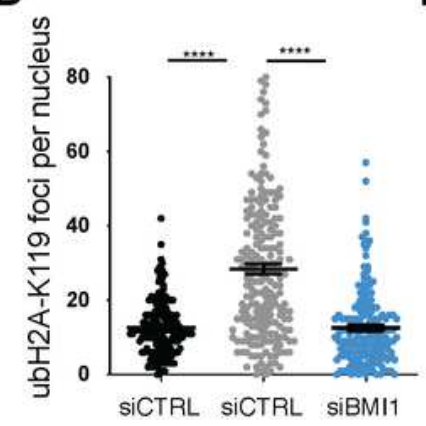

BMI1 controls DISC and histone ubH2A-K119 in DIvA cells. A. qRT-PCR analysis of the mRNA levels of six different break bearing genes upon treatment with siRNAs against BMI1 or ATM or non-targeting control siRNAs (siCTRL). Data are depicted relative to uncut cells for each knockdown condition. Transcript level of each gene in uncut condition has been set to one. Error bars represent SEM of fold change of 18 transcripts from 3 independent experiments. $P<0.0001(\star \star \star \star)$.Statistical test used was One-Way ANOVA. 
B. Representative images of $\mathrm{YH} 2 \mathrm{AX}$ and $\mathrm{ubH} 2 \mathrm{~A}-\mathrm{K} 119$ foci in cut and uncut cells treated with siRNAs against BMI1 or ATM or non-targeting control siRNAs (siCTRL). DNA was stained by DAPI. Scale bars: 20 $\mu \mathrm{m}$. C. Quantification of $\mathrm{YH} 2 \mathrm{AX}$ foci in cut and uncut cells treated with non-targeting control siRNAs or siRNAs specific for BMI1. Error bars represent SEM from more than 150 cells from two independent experiments. D. Quantification of ubH2A-K119 foci in cut and uncut cells treated with non-targeting control siRNAs or siRNAs specific for BMI1. Error bars represent SEM from more than 150 cells from two independent experiments. $P<0.0001$. Statistical test used was One-Way ANOVA E. Western Blot analysis of BMI1 protein levels in uncut and cut cells treated with non-targeting control siRNAs or siRNAs against BMI1. Actin immunoblot was used as loading control. 
Figure 4

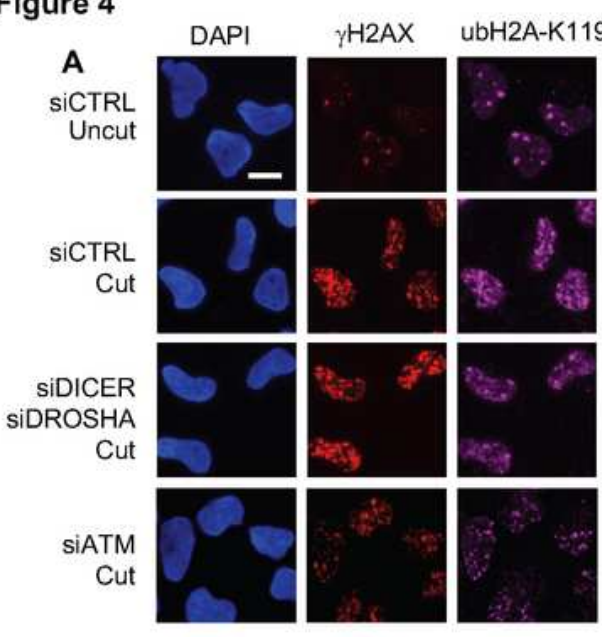

D
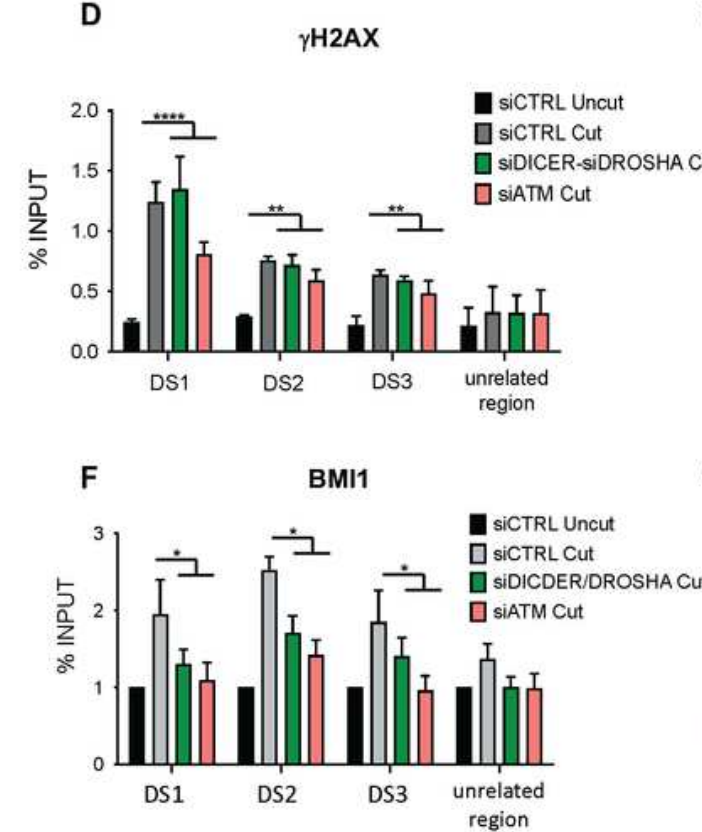

H

BMI1-DI-PLA

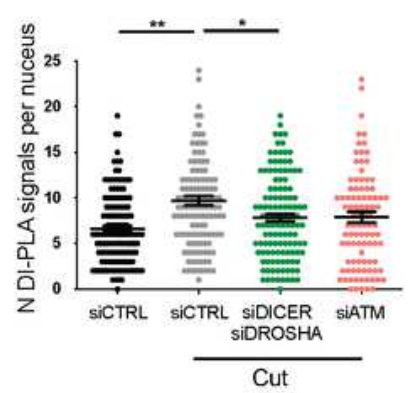

B

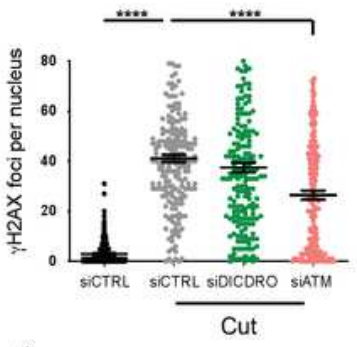

C

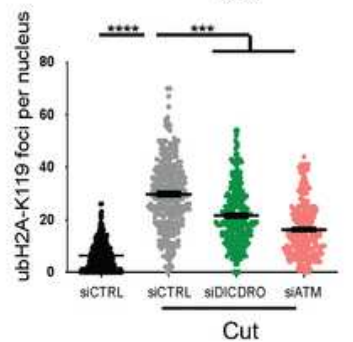

E

ubH2A-K119

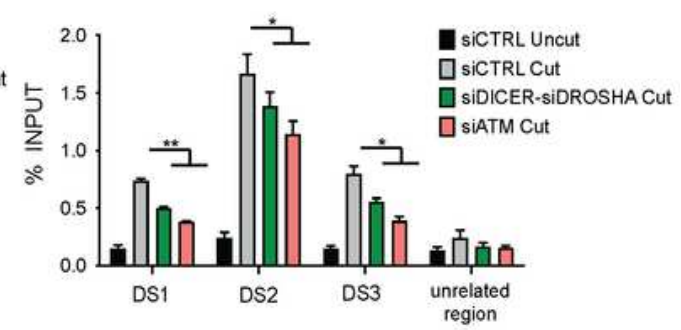

G

BMI1-DI-PLA

Uncut

$\begin{array}{llll} & \text { SICTRL } & \text { SiCTRLER } & \text { SIDROSHA } \\ \text { SIDTM }\end{array}$
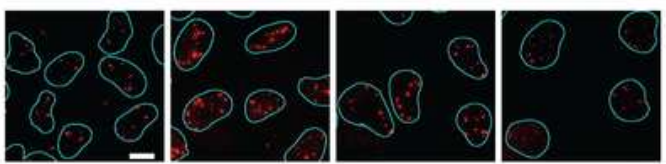

Figure 4

DICER and DROSHA inactivation also reduces ubH2A-K119 foci and BMI1 recruitment at DNA damage sites. A. Representative images of $\mathrm{YH} 2 \mathrm{AX}$ and $\mathrm{ubH} 2 \mathrm{~A}-\mathrm{K} 119$ foci in cut and uncut cells treated with nontargeting control siRNAs (SICTRL) or siRNAs against DICER and DROSHA or ATM. DNA was stained by DAPI. Scale bars: $20 \mu \mathrm{m}$. B. Quantification of $\mathrm{yH} 2 \mathrm{AX}$ foci in cut and uncut cells treated as in A. Error bars represent SEM from more than 100 cells from two independent experiments. $P<0.0001$. C. Quantification 
of ubH2A-K119 foci in cut and uncut cells treated as in A. Error bars represent SEM from more than 100 cells from two independent experiments. $\left.\mathrm{P}<0.001{ }^{(\star \star \star}\right)$ or $\left.\mathrm{P}<0.0001{ }^{(\star \star \star \star}\right)$. D-F. ChIP-qPCR analysis at three AsiSI cut sites and an unrelated region in uncut and cut cells treated with non-targeting control siRNAs or siRNAs for DICER and DROSHA or ATM for the markers YH2AX (D), ubH2A-K119 (E) and BMI1 protein $(F)$. Error bars represent SEM from 3 independent experiments. $P<0.0001$ ( $\left.{ }^{* \star *}\right), P<0.01$ ( $\left.{ }^{* *}\right)$ or $\left.\mathrm{P}<0.05{ }^{(}\right)$. G. Representative images of DI-PLA signals for BMI1 recruitment to sites of damage in cut and uncut cells treated with non-targeting control siRNAs or siRNA specific for DICER and DROSHA or ATM. H. Quantification of DI-PLA signals for BMI1 recruitment in cut) and uncut cells treated with nontargeting control siRNAs or siRNAs specific for DICER and DROSHA or ATM. Error bars represent SEM from more than 100 cells from three independent experiments. $P<0.05\left(^{*}\right)$ or $P<0.01(* *)$. Statistical test used was One-Way ANOVA. 


\section{Figure 5}

A

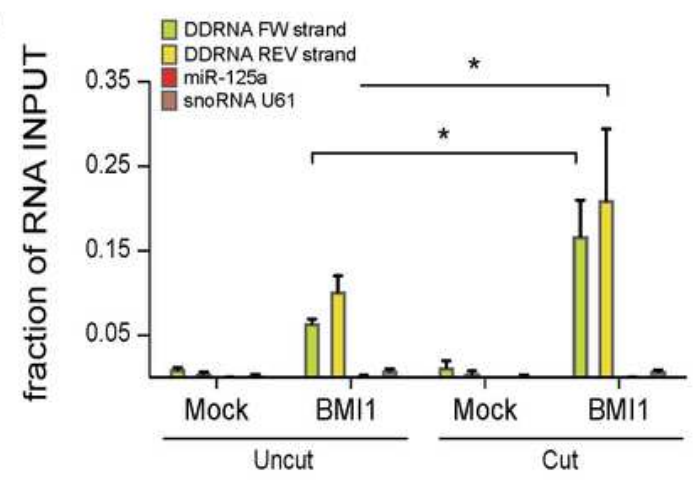

C
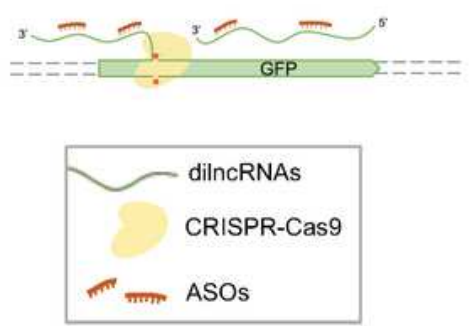

B

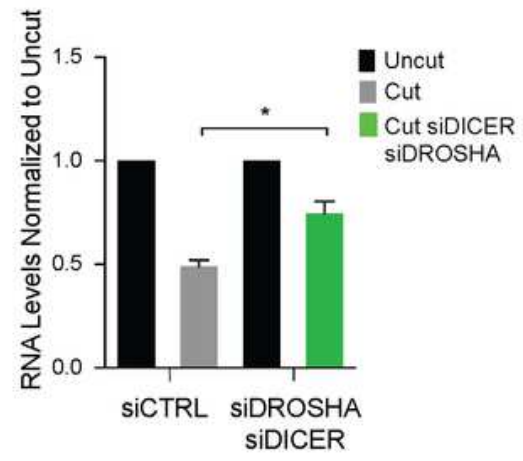

D

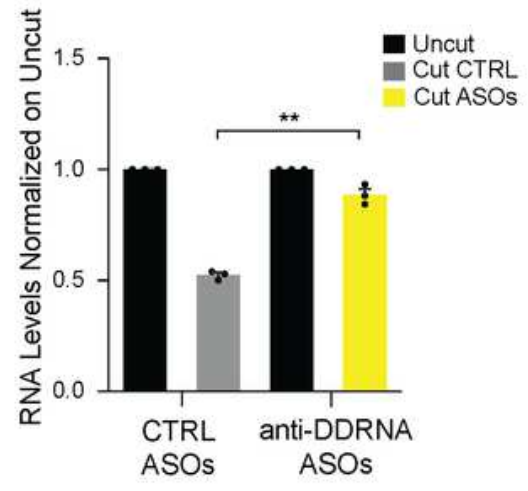

Figure 5

At sites of damage BMI1 interacts with DDRNAs, and transfecting ASOs inhibiting DDRNA is sufficient to abolish DISC. A. RNA immunoprecipitation (RIP) analysis of BMI1 followed by miScript RT II retrotranscription and qPCR analysis to detect association with DDRNAs (or miR-125a as control) in NIH2/4 cut and uncut cells. Error bars represent SEM from 3 independent experiments. $P<0.05$. Statistical test used was Two-Way ANOVA. B. qRT-PCR analysis of GFP mRNA levels in cut cells relative to uncut ones 
set to one in HeLa cells with an integrated GFP gene (HeLa-GFP) treated with control siRNAs SiCTRL or siRNAs against DROSHA and DICER. Error bars represent SEM from 3 independent experiments. $P<0.05$. Statistical test used was paired Student t-test. C Schematic representation of the construct integrated in HeLa-GFP used to evaluate gene expression following CRISPR-Cas9 cleavage. ASOs: sequence-specific antisense oligonucleotides. D. qRT-PCR analysis of GFP mRNA levels in cut and uncut cells treated with non-targeting control ASOs or ASOs specific for the dilncRNAs generated at this locus. Error bars represent SEM from 3 independent experiments. Data are depicted relative to uncut cells. $P<0.01$. Statistical test used was paired Student t-test.

\section{Supplementary Files}

This is a list of supplementary files associated with this preprint. Click to download.

- SupplementaryFigures.pdf

- GEOaccessionnumberforRNAsequencingdata.pdf

- Francianrreportingsummaryflatten.pdf 